\title{
Power Transport Theorem Based Decoupling Mode Theory for Wave-Port-Fed Transmitting Antennas
}

This paper was downloaded from TechRxiv (https://www.techrxiv.org).

\section{LICENSE}

CC BY-NC-SA 4.0

SUBMISSION DATE / POSTED DATE

09-09-2021 / 11-09-2021

CITATION

Lian, Renzun; Xia, Mingyao (2021): Power Transport Theorem Based Decoupling Mode Theory for WavePort-Fed Transmitting Antennas. TechRxiv. Preprint. https://doi.org/10.36227/techrxiv.16592702.v1

DOI

10.36227/techrxiv.16592702.v1 


\title{
Power Transport Theorem Based Decoupling Mode Theory for Wave-Port-Fed Transmitting Antennas
}

\author{
Ren-Zun Lian and Ming-Yao Xia, Senior Member, IEEE
}

\begin{abstract}
Employing the work-energy principle based physical interpretation for characteristic mode theories (CMTs), it is exposed that: strictly speaking, the existing CMTs are the modal analysis theories for incident-field-driven scattering objects and lumped-port-driven transmitting antennas, but not for wave-port-fed transmitting antennas, so they cannot provide an exact modal analysis to wave-port-fed antennas. This paper focuses on establishing an effective modal analysis theory for wave-port-fed antennas. Power transport theorem (PTT), which governs the transport process of the power-flow passing through wave-port-fed antenna, is derived. It is found out that the input power contained in PTT is the source to sustain a stationary power transport. Under PTT framework, a novel modal analysis theory - decoupling mode theory (DMT) - is established for the antenna. By orthogonalizing input power operator (IPO), the PTT-based DMT can construct a set of energy-decoupled modes (DMs) for the antenna. A novel concept of "electric-magnetic energy-decoupling factor" $/$ "electric-magnetic phase-mismatching factor" is introduced for quantifying the coupling/matching degree between modal electric field and modal magnetic field. A field-based definition for modal input impedance and admittance is proposed as an alternative for the conventional circuit-based definition. Three somewhat different but not contradictory physical meanings of modal significance (MS) are summarized.
\end{abstract}

Index Terms-Decoupling mode theory (DMT), energy-decoupled mode (DM), input power operator (IPO), power transport theorem (PTT), wave-port-fed transmitting antenna.

\section{INTRODUCTION}

$\mathrm{F}$ OR ANY linear electromagnetic (EM) structure (either scattering object or transmitting antenna or others), its all physically realizable working modes constitute a linear space [1]-[2]. The one-by-one analysis for the modes is impracticable, because the number of the modes is infinite. A practicable way is to analyze the basis of the linear space (i.e., the fundamental working modes of the EM structure), because any mode can be expanded in terms of the fundamental modes [3]-[4]. For a certain EM structure, it has many different fundamental mode sets. Among the various fundamental mode sets, the sets constituted by orthogonal modes are especially important [1]-[4].

Characteristic modes (CMs) are a series of orthogonal modes,

Manuscript with identifier [AP2104-0777] was submitted to IEEE-TAP on April 14, 2021. (Corresponding authors: Ren-Zun Lian; Ming-Yao Xia.)

R. Z. Lian and M. Y. Xia are with the Department of Electronics, School of Electronics Engineering and Computer Science, Peking University, Beijing 100871, China. (e-mail: rzlian@vip.163.com; myxia@pku.edu.cn). and they were first introduced by Garbacz et al. [5]-[7] in the 1960s. Garbacz et al. established their characteristic mode theory (CMT), or called characteristic mode analysis (CMA), under scattering matrix (SM) framework, and the SM-based CMT (SM-CMT) constructs CMs by orthogonalizing perturbation matrix operator (PMO). In the 1970s, Harrington et al. [8]-[12] developed an alternative way to establish CMT under integral equation (IE) framework, and used the IE-based CMT (IE-CMT) to construct CMs by orthogonalizing impedance matrix operator (IMO). Recently, [13]-[15] rebuilt Harrington's IE-CMT in a novel work-energy principle (WEP) framework, and the WEP-based CMT (WEP-CMT) constructs CMs by orthogonalizing driving power operator (DPO).

IE-CMT has been used to do approximate modal analysis for some kinds of transmitting antennas (such as circularly polarized [16]-[17], reconfigurable [18]-[20], wearable [21], RFID tag [22]-[23], direction-finding [24], and multimode [25]-[26] antennas), and some typical IE-CMT-based antenna applications have been summarized in [27]-[34]. But, using the physical interpretation obtained in [13]-[15], recently [35] and [36, Sec. 1.3] pointed out that both the IE-CMT [8]-[12] and WEP-CMT [13]-[15] are inapplicable to Yagi-Uda transmitting antenna arrays (a typical metallic Yagi-Uda array is shown in Fig. 1(a)), and the physical explanations for the inapplicability are as below.

- The CMs constructed in [8]-[15] orthogonalize DPO, i.e., the power done by incident fields on scattered currents, so the corresponding CMTs are the theories for incident-field-driven scattering objects as shown in Fig. 2.

- The working mechanism of transmitting antennas is different from the working mechanism of scattering objects.

- As shown in Fig. 1, transmitting antenna is a device or circuit that generates high-frequency electric energy, controlled or modulated, which can be emitted from a finite region in the form of unguided waves [37, pp. 369 and pp. 1210].

- As shown in Fig. 2, scattering object is a secondary structure generating scattered fields resulted from the scattered currents induced on the structure by some fields incident on the structure from some primary sources $[37$, pp. 1006].

Thus, strictly speaking the CMTs built in [8]-[15] are not the rigorous theories for transmitting antennas, though they have been approximately applied to some antennas.

- Yagi-Uda arrays belong to the family of transmitting antennas rather than the family of scattering objects. 


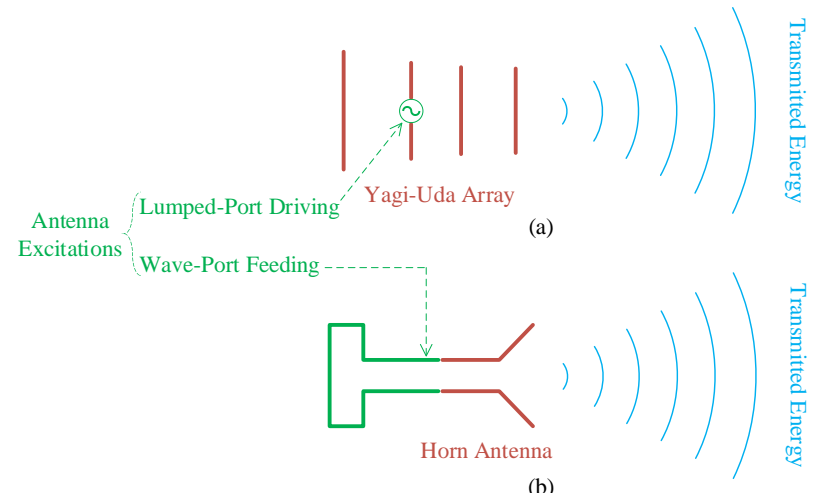

Fig. 1. Two typical transmitting antennas radiating EM energies. (a) A lumped-port-driven Yagi-Uda array; (b) a wave-port-fed horn antenna.

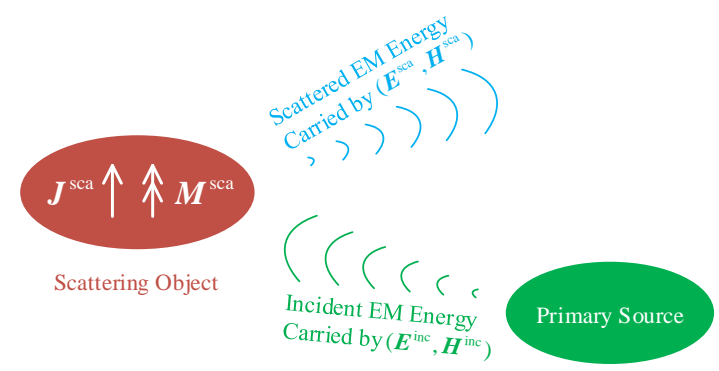

Fig. 2. A typical scattering object driven by externally incident field.

To do the modal analysis for Yagi-Uda arrays, [35] and [36, Sec. 4.2] developed an alternative WEP-CMT for Yagi-Uda arrays, which theory is different from the scattering-object -oriented CMTs given in [8]-[15]. Afterwards, [36, Sec. 4.6] and [38] further generalized the WEP-CMT for Yagi-Uda arrays to the WEP-CMT for wireless power transfer systems. The evolution relations about the various CMTs mentioned above are summarized in Tab. I.

In fact, besides Yagi-Uda arrays, there also exist many classical antennas to which the CMTs established in [8]-[15] cannot be applied, such as the metallic horn antenna shown in Fig. 1(b). For the horn proposed in [41, Sec. 3.4.3] and shown in Fig. 3(a), its desired mode working at $11.70 \mathrm{GHz}$ has the far-field radiation pattern shown in Fig. 3(b). The modal significances (MSs) associated to the first several lower-order IE-based CMs (IE-CMs) of the horn are shown in Fig. 4(a), and the radiation patterns of the lower-order IE-CMs working at $11.70 \mathrm{GHz}$ are shown in Fig. 4(b). Evidently, all the IE-CMs are not consistent with the desired mode reported in [41, Sec. 3.4.3].

Moreover, besides the IE-CMT [8]-[12] and WEP-CMT [13]-[15] for scattering objects, the WEP-CMT for Yagi-Uda arrays [35], [36, Sec. 4.2] is also inapplicable to the horn, because the excitation mechanism for Yagi-Uda arrays and the excitation mechanism for horns are essentially different from each other. Specifically,

- the Yagi-Uda array is driven by a lumped-port excitation (e.g. delta-gap voltage source) as shown in Fig. 1(a), but

- the horn antenna is fed by a wave-port excitation (e.g. waveguide transmission line) as shown in Fig. 1(b).

The above observations naturally expose a major problem and challenge in Antennas \& Propagation Society: how to establish an effective modal analysis theory for wave-port-fed transmitting antennas?

The central purpose of this paper is to resolve the above problem, and this paper is organized as follows: under a new power transport theorem (PTT) framework, Sec. II establishes a novel modal analysis theory - decoupling mode theory (DMT) - for wave-port-fed metallic antennas; Sec. III generalizes the PTT-based DMT (PTT-DMT) to wave-port-fed material antennas; Sec. IV concludes this paper; some trivial mathematical details are listed in the appendix. The $e^{j \omega t}$ convention and inner product $<\boldsymbol{f}, \boldsymbol{g}>_{\Omega}=\int_{\Omega} \boldsymbol{f}^{\dagger} \cdot \boldsymbol{g} d \Omega$ are used throughout, where " $\dagger$ " is conjugate transpose operation, and the frequency-domain and time-domain fields are denoted as $\boldsymbol{F}$ and $\mathcal{F}$.

TABLE I Evolution RELATIONS AMONG THE MODAL ANALYSIS THEORIES MENTIONED IN THIS PAPER

\begin{tabular}{|c|c|c|c|c|}
\hline & Objective Structure & Theoretical Formalism & Generating Operator & Modal Feature \\
\hline SM-CMT Established in [5]-[7] & $\begin{array}{l}\text { incident-field-driven } \\
\text { scattering object }\end{array}$ & $\begin{array}{l}\text { scattering matrix } \\
\quad(\mathrm{SM})\end{array}$ & $\begin{array}{l}\text { perturbation matrix operator } \\
\text { (PMO) }\end{array}$ & far-field decoupling \\
\hline \multicolumn{5}{|l|}{$\downarrow$} \\
\hline IE-CMT Established in [8]-[12] & same as above & $\begin{array}{l}\text { integral equation } \\
\text { (IE) }\end{array}$ & $\begin{array}{l}\text { impedance matrix operator } \\
\text { (IMO) }\end{array}$ & $\begin{array}{l}\text { not clarified } \\
\text { by its founders }\end{array}$ \\
\hline \multicolumn{5}{|l|}{$\downarrow$} \\
\hline WEP-CMT Established in [13]-[15] & same as above & $\begin{array}{l}\text { work-energy principle } \\
\text { (WEP) }\end{array}$ & $\begin{array}{l}\text { driving power operator } \\
\text { (DPO) }\end{array}$ & energy decoupling \\
\hline \multicolumn{5}{|l|}{$\downarrow$} \\
\hline $\begin{array}{c}\text { Alternative WEP-CMT Established in } \\
{[35],[36 \text {, Chap. 4], and [38] }}\end{array}$ & $\begin{array}{l}\text { lumped-port-driven } \\
\text { electromagnetic structures }\end{array}$ & same as above & same as above & same as above \\
\hline \multicolumn{5}{|l|}{$\downarrow$} \\
\hline PTT-DMT Established in This Paper & $\begin{array}{l}\text { wave-port-fed } \\
\text { transmitting antenna }\end{array}$ & $\begin{array}{l}\text { power transport theorem } \\
\text { (PTT) }\end{array}$ & $\begin{array}{l}\text { input power operator } \\
\text { (IPO) }\end{array}$ & same as above \\
\hline \multicolumn{5}{|l|}{$\downarrow$} \\
\hline $\begin{array}{l}\text { PTT-DMT for Waveguides Established in } \\
\text { [36, Sec. 3.4], [39], and [40, Chap. 3] }\end{array}$ & $\begin{array}{c}\text { wave-port-fed } \\
\text { wave-guiding structure }\end{array}$ & same as above & same as above & same as above \\
\hline
\end{tabular}



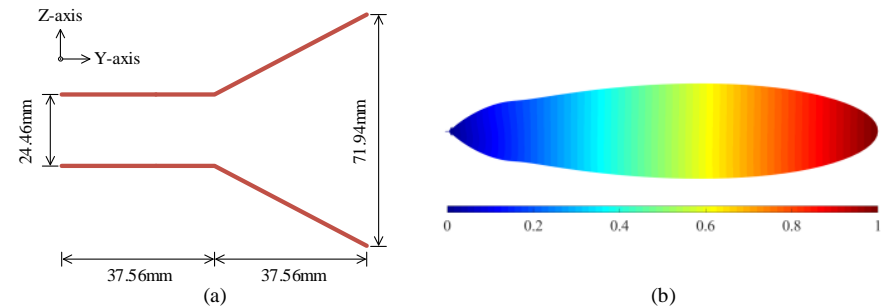

(b)

Fig. 3. (a) Size of the circular metallic horn antenna reported in [41, Sec. 3.4.3] and (b) radiation pattern of the desired mode (working at $11.70 \mathrm{GHz}$ ) of the horn.

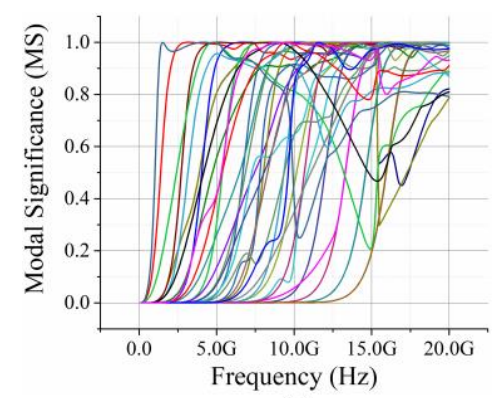

(a)
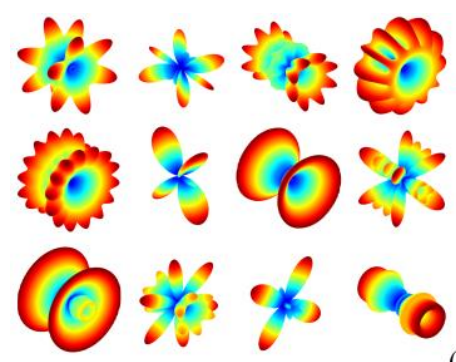

(a)
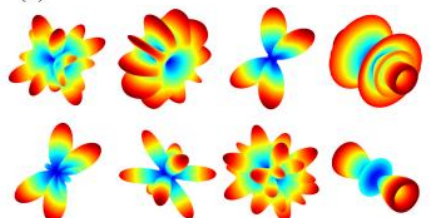

(b)

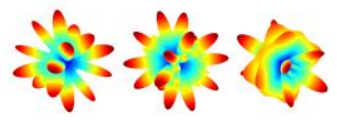

Fig. 4. (a) MSs associated to the first several lower-order IE-CMs calculated from the conventional IE-CMT established by Harrington et al. in [8]-[10] and (b) radiation patterns of the first several lower-order IE-CMs working at 11.7 GHz.

In addition, in our future study we will further generalize the idea of this paper to wave-guiding structures (i.e., waveguides). The evolution relations among the various DMTs and the various CMTs mentioned above are also illustrated in Tab. I.

\section{PTT-DMT For WAVE-Port-Fed Metallic Antennas}

Taking the metallic horn antenna shown in Fig. 5 as a typical example, this section establishes the PTT-DMT for wave-port-fed metallic transmitting antennas. The horn is placed in free-space environment $V^{\mathrm{e}}$ with material parameters $\left(\mu_{0}, \varepsilon_{0}\right)$ and outer boundary $S^{\infty}$. The input port (i.e., feeding port) and electric wall of the horn are denoted as $S^{\mathrm{i}}$ and $S^{\mathrm{e}}$ respectively. The normal direction of $S^{\mathrm{i}}$ is denoted as $\boldsymbol{n}$, and $\boldsymbol{n}$ points to $V^{\mathrm{e}}$ as shown in Fig. 5 .

\section{A. Physical Framework}

The conservation law of energy tells us that: if energy goes into or away from a region, it is because it flows into or away through the boundaries of that region [42, Sec. 27-1]. Employing Maxwell's equations, the conservation law governing the EM energies passing through the antenna shown in Fig. 5 can be quantitatively expressed as follows:

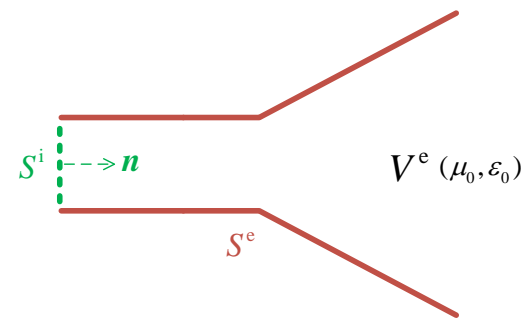

Fig. 5. Topological structure of a typical metallic horn antenna.

$$
P^{\text {in }}=P^{\text {rad }}+j 2 \omega\left(W^{\text {mag }}-W^{\text {ele }}\right)
$$

in which

$$
P^{\text {in }}=(1 / 2) \iint_{S^{\mathrm{i}}}\left(\boldsymbol{E} \times \boldsymbol{H}^{\dagger}\right) \cdot d \boldsymbol{S}
$$

and $P^{\mathrm{rad}}=(1 / 2) \iint_{S^{\infty}}\left(\boldsymbol{E} \times \boldsymbol{H}^{\dagger}\right) \cdot d \boldsymbol{S}$, and $W^{\mathrm{mag}}=(1 / 4)<\boldsymbol{H}, \boldsymbol{B}>_{V^{\mathrm{e}}}$, and $W^{\text {ele }}=(1 / 4)<\boldsymbol{D}, \boldsymbol{E}>_{V^{\mathrm{e}}}$, where $\boldsymbol{B}=\mu_{0} \boldsymbol{H}$ and $\boldsymbol{D}=\varepsilon_{0} \boldsymbol{E}$.

Relation (1) has a very clear physical interpretation: the input power $P^{\text {in }}$ inputted into the horn is transformed into two parts - radiated power $P^{\mathrm{rad}}$ carried by EM field from horn to infinity and reactive power $2 \omega\left(W^{\mathrm{mag}}-W^{\text {ele }}\right)$ used to contribute the energies stored in environment. Because (1) rigorously governs the transport process of the power-flow passing through the horn, then it is called power transport theorem $(P T T)$ in this paper. In fact, the PTT can be viewed as a generalization for famous Poynting's theorem (PtT) [36, Chap. 2], [40, Chap. 2].

The power term $P^{\text {in }}$ in (1) is just the source to sustain a stationary power transport, and its operator expression (2) is called input power operator (IPO). By orthogonalizing the IPO, PTT-DMT can construct a set of orthogonal modes satisfying the following power-decoupling relation on input port.

$$
(1 / 2) \iint_{S^{i}}\left(\boldsymbol{E}_{m} \times \boldsymbol{H}_{n}^{\dagger}\right) \cdot d \boldsymbol{S}=\left(1+j \theta_{m}\right) \delta_{m n}
$$

The physical meaning of the above $\theta_{m}$ will be carefully explained in Sec. II-C. In addition, the power-decoupling relation (3) also implies the following energy-decoupling relation

$$
(1 / T) \int_{t_{0}}^{t_{0}+T}\left[\iint_{S^{i}}\left(\mathcal{E}_{m} \times \mathcal{H}_{n}\right) \cdot d \boldsymbol{S}\right] d t=\delta_{m n}
$$

(or called time-average power-decoupling relation) and the following Parseval's identity

$$
(1 / T) \int_{t_{0}}^{t_{0}+T}\left[\iint_{S^{i}}(\mathcal{E} \times \mathcal{H}) \cdot d \boldsymbol{S}\right] d t=\sum_{m}\left|c_{m}\right|^{2}
$$

where $t_{0}$ is an arbitrary time point, and $T$ is the time period, and $\left\{c_{m}\right\}$ are the modal expansion coefficients used to expand the previously known field $(\mathcal{E}, \mathcal{H})$ in terms of the DMs.

Relations (1)-(5) clearly reveal the physical purpose of the modal analysis theory - to construct a set of energy-decoupled modes (DMs) for a wave-port-fed transmitting antenna. Just because of this physical purpose, the modal analysis theory is called decoupling mode theory $(D M T)$ in this paper. 


\section{B. Mathematical Formulations}

If the equivalent surface electric and magnetic currents on $S^{\mathrm{i}}$ are denoted as $\boldsymbol{J}^{\mathrm{i}}$ and $\boldsymbol{M}^{\mathrm{i}}$ respectively, and the induced surface electric current on $S^{\mathrm{e}}$ is denoted as $\boldsymbol{J}^{\mathrm{e}}$, then the IPO (2) can be rewritten as the following alternative forms

$$
\begin{aligned}
P^{\mathrm{in}} & =-(1 / 2)\left\langle\boldsymbol{J}^{\mathrm{i}},-j \omega \mu_{0} \mathcal{L}_{0}\left(\boldsymbol{J}^{\mathrm{i}}+\boldsymbol{J}^{\mathrm{e}}\right)-\mathcal{K}_{0}\left(\boldsymbol{M}^{\mathrm{i}}\right)\right\rangle_{S_{+}^{\mathrm{i}}} \\
& =-(1 / 2)\left\langle\boldsymbol{M}^{\mathrm{i}}, \mathcal{K}_{0}\left(\boldsymbol{J}^{\mathrm{i}}+\boldsymbol{J}^{\mathrm{e}}\right)-j \omega \varepsilon_{0} \mathcal{L}_{0}\left(\boldsymbol{M}^{\mathrm{i}}\right)\right\rangle_{S_{+}^{\mathrm{i}}}^{\dagger}
\end{aligned}
$$

where integral surface $S_{+}^{\mathrm{i}}$ is the right-side surface of $S^{\mathrm{i}}$. To effectively distinguish the two forms of $P^{\text {in }}$ in (6), the right-hand sides of the first and second equalities are called JE and HM interaction forms respectively.

By expanding the above-mentioned currents in terms of some proper basis functions, integral operators (6) can be discretized into the following matrix forms

$$
P^{\text {in }}=\left(\mathrm{J}^{\mathrm{i}}\right)^{\dagger} \cdot \mathrm{P}_{\mathrm{JE}} \cdot\left[\begin{array}{l}
\mathrm{J}^{\mathrm{i}} \\
\mathrm{J}^{\mathrm{e}} \\
\mathrm{M}^{\mathrm{i}}
\end{array}\right]=\left[\begin{array}{l}
\mathrm{J}^{\mathrm{i}} \\
\mathrm{J}^{\mathrm{e}} \\
\mathrm{M}^{\mathrm{i}}
\end{array}\right]^{\dagger} \cdot \mathrm{P}_{\mathrm{HM}} \cdot \mathrm{M}^{\mathrm{i}}
$$

where $\mathrm{J}^{\mathrm{i}}, \mathrm{J}^{\mathrm{e}}$, and $\mathrm{M}^{\mathrm{i}}$ are the basis function expansion vectors of $\boldsymbol{J}^{\mathrm{i}}, \boldsymbol{J}^{\mathrm{e}}$, and $\boldsymbol{M}^{\mathrm{i}}$ respectively.

In fact, currents $\boldsymbol{J}^{\mathrm{i}}, \boldsymbol{J}^{\mathrm{e}}$, and $\boldsymbol{M}^{\mathrm{i}}$ are not independent, because they satisfy the following integral equations

$$
\begin{aligned}
& {\left[\mathcal{K}_{0}\left(\boldsymbol{J}^{\mathrm{i}}+\boldsymbol{J}^{\mathrm{e}}\right)-j \omega \varepsilon_{0} \mathcal{L}_{0}\left(\boldsymbol{M}^{\mathrm{i}}\right)\right]_{S_{+}^{\mathrm{i}}}^{\mathrm{tan}}=\boldsymbol{J}^{\mathrm{i}} \times \boldsymbol{n}} \\
& {\left[-j \omega \mu_{0} \mathcal{L}_{0}\left(\boldsymbol{J}^{\mathrm{i}}+\boldsymbol{J}^{\mathrm{e}}\right)-\mathcal{K}_{0}\left(\boldsymbol{M}^{\mathrm{i}}\right)\right]_{S_{+}^{\mathrm{i}}}^{\mathrm{tan}}=\boldsymbol{n} \times \boldsymbol{M}^{\mathrm{i}}} \\
& {\left[-j \omega \mu_{0} \mathcal{L}_{0}\left(\boldsymbol{J}^{\mathrm{i}}+\boldsymbol{J}^{\mathrm{e}}\right)-\mathcal{K}_{0}\left(\boldsymbol{M}^{\mathrm{i}}\right)\right]_{S^{\mathrm{e}}}^{\tan }=0}
\end{aligned}
$$

Here, (8) and (9) are based on the definitions of $\boldsymbol{J}^{\mathrm{i}}$ (DoJ) and $\boldsymbol{M}^{\mathrm{i}}$ (DoM) respectively, and (10) is based on the homogeneous tangential electric field boundary condition on $S^{\mathrm{e}}$.

Applying the method of moments (MoM) to (8)-(10), the integral equations are immediately discretized into some matrix equations. By solving the matrix equations, it is easy to derive the transformations from independent current $\mathrm{J}^{\mathrm{i}} / \mathrm{M}^{\mathrm{i}}$ into dependent currents $\left(\mathrm{J}^{\mathrm{e}}, \mathrm{M}^{\mathrm{i}}\right) /\left(\mathrm{J}^{\mathrm{i}}, \mathrm{J}^{\mathrm{e}}\right)$, and then the following

$$
\left[\begin{array}{l}
J^{i} \\
J^{e} \\
M^{i}
\end{array}\right]=T_{\text {DoJ }} \cdot J^{i}=T_{\text {DoM }} \cdot M^{i}
$$

from independent current $\mathrm{J}^{\mathrm{i}} / \mathrm{M}^{\mathrm{i}}$ into all currents $\left(\mathrm{J}^{\mathrm{i}}, \mathrm{J}^{\mathrm{e}}, \mathrm{M}^{\mathrm{i}}\right)$. $\mathrm{J}^{\mathrm{i}}, \mathrm{J}^{\mathrm{e}}$, and $\mathrm{M}^{\mathrm{i}}$ are the current expansion coefficient vectors.

Substituting transformations (11) into matrix forms (7), the

$$
P^{\text {in }}=\left(\mathrm{C}^{\mathrm{i}}\right)^{\dagger} \cdot \mathrm{P}^{\text {in }} \cdot \mathrm{C}^{\mathrm{i}}=\left\{\begin{array}{l}
\left(\mathrm{J}^{\mathrm{i}}\right)^{\dagger} \cdot \overbrace{\mathrm{P}_{\mathrm{JE}} \cdot \mathrm{T}_{\text {DOJ }}^{\mathrm{P}_{\mathrm{JE}} \text { DoJ }}}^{\mathrm{T}} \cdot \mathrm{J}^{\mathrm{i}} \\
\left(\mathrm{M}^{\mathrm{i}}\right)^{\dagger} \cdot \underbrace{\mathrm{T}_{\text {DoM }}^{\dagger} \cdot \mathrm{P}_{\mathrm{HM}}}_{\mathrm{P}_{\text {HM-DOM }}} \cdot \mathrm{M}^{\mathrm{i}}
\end{array}\right.
$$

with only independent current $\mathrm{C}^{\mathrm{i}}$ (which is either $\mathrm{J}^{\mathrm{i}}$ or $\mathrm{M}^{\mathrm{i}}$ ) is obtained. Employing the $\mathrm{P}^{\text {in }}$ in (12), the DMs can be derived from solving the following modal decoupling equation

$$
\mathrm{P}_{-}^{\mathrm{in}} \cdot \mathrm{C}_{m}^{\mathrm{i}}=\theta_{m} \mathrm{P}_{+}^{\mathrm{in}} \cdot \mathrm{C}_{m}^{\mathrm{i}}
$$

with $\mathrm{P}_{+}^{\text {in }}=\left[\mathrm{P}^{\text {in }}+\left(\mathrm{P}^{\text {in }}\right)^{\dagger}\right] / 2$ and $\mathrm{P}_{-}^{\text {in }}=\left[\mathrm{P}^{\text {in }}-\left(\mathrm{P}^{\text {in }}\right)^{\dagger}\right] / 2 j$.

Because the DMs are complete, any working mode $\mathrm{C}^{\mathrm{i}}$ can be linearly expanded in terms of the DMs as follows:

$$
\mathrm{C}^{\mathrm{i}}=\sum_{m} c_{m} \mathrm{C}_{m}^{\mathrm{i}}
$$

in which expansion coefficients $\left\{c_{m}\right\}$ have explicit expression

$$
c_{m}=\frac{(1 / 2) \iint_{S^{i}}\left(\boldsymbol{E} \times \boldsymbol{H}_{m}^{\dagger}\right) \cdot d \boldsymbol{S}}{1+j \theta_{m}}=\frac{(1 / 2) \iint_{S^{i}}\left(\boldsymbol{E}_{m} \times \boldsymbol{H}^{\dagger}\right) \cdot d \boldsymbol{S}}{1+j \theta_{m}}(15)
$$

due to the modal decoupling relation (3), where fields $(\boldsymbol{E}, \boldsymbol{H})$ are previously known.

\section{Modal Quantities}

This sub-section focuses on discussing some useful scalar quantities used to quantitatively depict the modal features in the aspects of utilizing EM energies.

\section{Modal Electric-Magnetic Energy-Decoupling Factor / Modal Electric-Magnetic Phase-Mismatching Factor / Modal @-Factor}

By generalizing the ideas of [13, Sec.3.3], [14], and [43], all the DMs $\left\{\mathrm{C}_{m}^{\mathrm{i}}\right\}$ can be classified into three categories - purely inductive DMs $\left\{\mathrm{C}_{\zeta}^{\text {ind }}\right\}$ (whose modal imaginary powers are positive), purely resonant DMs $\left\{\mathrm{C}_{s}^{\text {res }}\right\}$ (whose modal imaginary powers are zero), and purely capacitive DMs $\left\{\mathrm{C}_{\xi}^{\text {cap }}\right\}$ (whose modal imaginary powers are negative), and then any working mode $\mathrm{C}^{\mathrm{i}}$ can be decomposed as follows:

$$
\mathrm{C}^{\mathrm{i}}=\underbrace{\sum_{\zeta} c_{\zeta}^{\text {ind }} \mathrm{C}_{\zeta}^{\text {ind }}}_{\mathrm{C}^{\text {ind }}}+\underbrace{\sum_{\zeta} c_{\zeta}^{\text {res }} \mathrm{C}_{\zeta}^{\text {res }}}_{\mathrm{C}^{\text {res }}}+\underbrace{\sum_{\xi} c_{\xi}^{\text {cap }} \mathrm{C}_{\xi}^{\text {cap }}}_{\mathrm{C}^{\text {aap }}}
$$

where $\mathrm{C}^{\text {ind }}, \mathrm{C}^{\text {res }}$, and $\mathrm{C}^{\text {cap }}$ are respectively called purely inductive component, purely resonant component, and purely capacitive component of the mode $\mathrm{C}^{\mathrm{i}}$.

Based on the above modal decomposition, we introduce the following modal quantity

$$
\begin{aligned}
\Theta\left(\mathrm{C}^{\mathrm{i}}\right) & =\frac{\overbrace{\left(\mathrm{C}^{\text {ind }}\right)^{\dagger} \cdot \mathrm{P}_{-}^{\text {in }} \cdot \mathrm{C}^{\text {ind }}}^{\text {this term is positive }}-\overbrace{\left(\mathrm{C}^{\text {cap }}\right)^{\dagger} \cdot \mathrm{P}_{-}^{\text {in }} \cdot \mathrm{C}^{\text {cap }}}^{\text {this term is negative }}}{\left(\mathrm{C}^{\mathrm{i}}\right)^{\dagger} \cdot \mathrm{P}_{+}^{\text {in }} \cdot \mathrm{C}^{\mathrm{i}}} \\
& =\frac{\left(\mathrm{C}^{\text {ind }}+j \mathrm{C}^{\text {cap }}\right)^{\dagger} \cdot \mathrm{P}_{-}^{\text {in }} \cdot\left(\mathrm{C}^{\text {ind }}+j \mathrm{C}^{\text {cap }}\right)}{\left(\mathrm{C}^{\mathrm{i}}\right)^{\dagger} \cdot \mathrm{P}_{+}^{\text {in }} \cdot \mathrm{C}^{\mathrm{i}}}
\end{aligned}
$$

The $\Theta\left(C^{\mathrm{i}}\right)$ quantitatively depicts "decoupling degree of modal electric and magnetic energies" and "mismatching degree of modal electric and magnetic phases". Clearly, the larger 
$\Theta\left(C^{i}\right)$ is, the stronger electric-magnetic energy-decoupling is, and the stronger electric-magnetic phase-mismatching is. Thus $\Theta\left(\mathrm{C}^{\mathrm{i}}\right)$ is called modal electric-magnetic energy-decoupling factor or modal electric-magnetic phase-mismatching factor in this paper, and simply denoted as modal $\Theta$-factor.

Obviously, for any single $\mathrm{DM} \mathrm{C}_{m}^{\mathrm{i}}$, there exists the following more simplified relation

$$
\Theta\left(\mathrm{C}_{m}^{\mathrm{i}}\right)=\left|\theta_{m}\right|
$$

and this relation clearly reveals the physical meaning of $\left|\theta_{m}\right|$ - $\Theta$-factor of the $m$-th DM itself.

Modal Input Impedance and Modal Input Admittance

For the DMs obtained above, we propose the following field-based definitions for modal input impedance and modal input admittance

$$
\begin{aligned}
& Z_{m}^{\text {in }}=\frac{(1 / 2) \iint_{S^{\mathrm{i}}}\left(\boldsymbol{E}_{m} \times \boldsymbol{H}_{m}^{\dagger}\right) \cdot d \boldsymbol{S}}{(1 / 2)\left\langle\boldsymbol{J}_{m}^{\mathrm{i}}, \boldsymbol{J}_{m}^{\mathrm{i}}\right\rangle_{S^{\mathrm{i}}}}=R_{m}^{\mathrm{in}}+j X_{m}^{\mathrm{in}} \\
& Y_{m}^{\mathrm{in}}=\frac{(1 / 2) \iint_{S^{\mathrm{i}}}\left(\boldsymbol{E}_{m} \times \boldsymbol{H}_{m}^{\dagger}\right) \cdot d \boldsymbol{S}}{(1 / 2)\left\langle\boldsymbol{M}_{m}^{\mathrm{i}}, \boldsymbol{M}_{m}^{\mathrm{i}}\right\rangle_{S^{\mathrm{i}}}}=G_{m}^{\mathrm{in}}+j B_{m}^{\mathrm{in}}
\end{aligned}
$$

where $R_{m}^{\text {in }}=\operatorname{Re}\left\{Z_{m}^{\text {in }}\right\}$ and $X_{m}^{\text {in }}=\operatorname{Im}\left\{Z_{m}^{\text {in }}\right\}$ are the corresponding modal input resistance and modal input reactance respectively, and $G_{m}^{\text {in }}=\operatorname{Re}\left\{Y_{m}^{\text {in }}\right\}$ and $B_{m}^{\text {in }}=\operatorname{Im}\left\{Y_{m}^{\text {in }}\right\}$ are the corresponding modal input conductance and modal input susceptance respectively.

The field-based definition can be treated as an alternative of the conventional circuit-based definition for impedance and admittance, and will be utilized in the following Sec. II-D.

\section{Modal Significance (MS)}

From Parseval's identity (5) and expansion coefficient (15), it is not difficult to find out that the following modal quantity

$$
\mathrm{MS}_{m}=\frac{1}{\left|1+j \theta_{m}\right|}
$$

which is traditionally called modal significance (MS), is a quantitative depiction for the weight of a single DM in whole modal expansion formulation (14).

In fact, the $\mathrm{MS}_{m}$ can also be alternatively written as the following equivalent forms

$$
\mathrm{MS}_{m}=\frac{R_{m}^{\text {in }}}{\left|R_{m}^{\text {in }}+j X_{m}^{\text {in }}\right|}=\frac{G_{m}^{\text {in }}}{\left|G_{m}^{\text {in }}+j B_{m}^{\text {in }}\right|}
$$

so the $\mathrm{MS}_{m}$ can also be physically interpreted as the weight of the modal resistance/conductance in whole modal impedanceladmittance.

In addition, it is clear that $\mathrm{MS}_{m}$ is a monotonously decreasing function about $\left|\theta_{m}\right|$, so: the larger $\mathrm{MS}_{m}$ is, the stronger electric-magnetic energy-coupling/phase-matching of the DM is. Thus, $\mathrm{MS}_{m}$ is also a quantitative depiction for the energy-coupling/phase-matching degree between the electric and magnetic fields of a single DM, just like the $\Theta$-factor $\theta_{m}$.

Now, we summarize the above three somewhat different but not contradictory physical interpretations for MS as follows:

1) MS is a quantitative depiction for the weight of a certain DM contained in whole modal expansion formulation;

2) MS is a quantitative depiction for the weight of the modal resistance/conductance in whole modal impedance/admittance;

3) MS is a quantitative depiction for the energy-coupling/phase-matching degree of the electric field and magnetic field carried by the single DM.

\section{Numerical Verifications}

In this subsection, the PTT-DMT-based modal analysis for two typical wave-port-fed metallic antennas are done for verifying the validity of the above theory and formulations.

\section{Metallic Horn Antenna}

Here, the circular metallic horn antenna reported in [41, Sec. 3.4.3] is analyzed, and its geometrical size is shown in Fig. 3(a). The JE-DoJ-based and HM-DoM-based modal resistance and modal significance (MS) curves are shown in Fig. 6. The figures imply that the JE-DoJ-based and HM-DoM-based results are consistent with each other, and DM 1 and DM 2 are resonant at frequency $7.4 \mathrm{GHz}$, and DM 3 and DM 4 are resonant at frequency $12.1 \mathrm{GHz}$, where "resonance" is defined as the working state at which $R^{\text {in }}$ attains its maximum [44, pp. 440].

The modal port currents of the resonant DMs 1 and 2 at 7.4 $\mathrm{GHz}$ are shown in Fig. 7, and the currents imply that the DMs are degenerate spatially (due to Y-axis rotational symmetry of the horn), and the modal radiation pattern and modal electric and magnetic fields of the first degenerate state are shown in Figs. 8 and 9, and the corresponding time-domain dynamic figures of the propagating fields are also uploaded to IEEE manuscript system together with this paper.

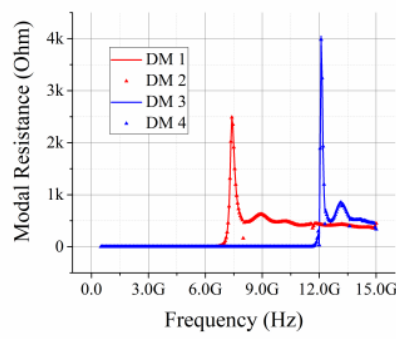

(a)

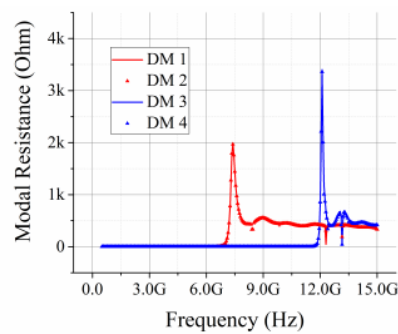

(c)

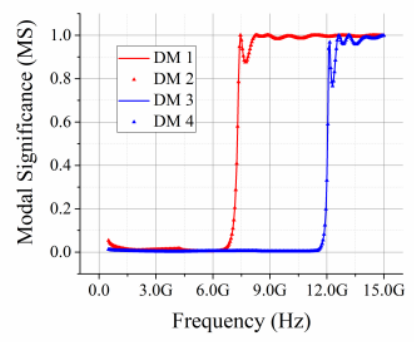

(b)

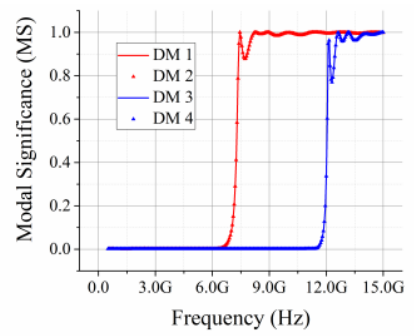

(d)
Fig. 6. (a) JE-DoJ-based modal resistances, (b) JE-DoJ-based modal significances, (c) HM-DoM-based modal resistances, and (d) HM-DoM-based modal significances of the first four lower-order DMs of the metallic horn antenna. 

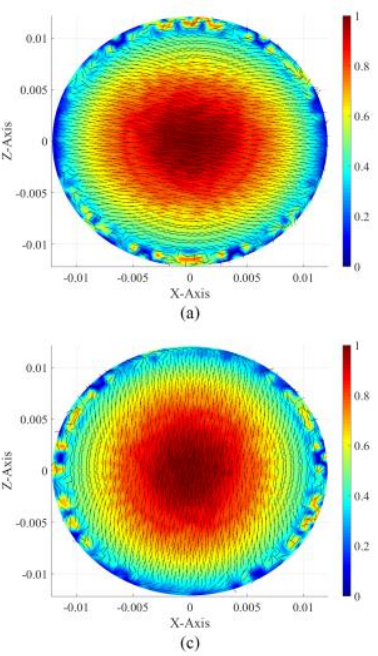

Fig. 7. (a) Modal port electric current of the resonant DM 1 at $7.4 \mathrm{GHz}$, (b) modal port magnetic current of the resonant DM 1 at $7.4 \mathrm{GHz}$, (c) modal port electric current of the resonant DM 2 at $7.4 \mathrm{GHz}$, and (d) modal port magnetic current of the resonant DM 2 at $7.4 \mathrm{GHz}$.

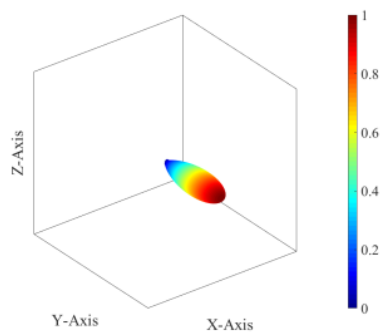

Fig. 8. Modal radiation pattern of the resonant DM 1 at $7.4 \mathrm{GHz}$.
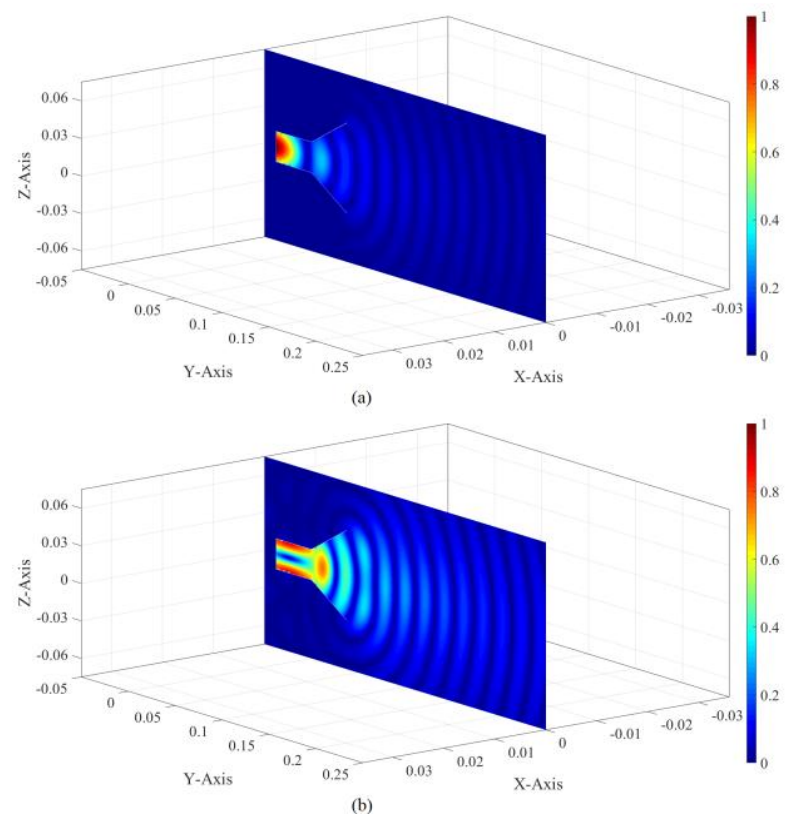

Fig. 9. Modal (a) electric and (b) magnetic field magnitude distributions of the resonant DM 1 at $7.4 \mathrm{GHz}$.

The modal port currents of the resonant DMs 3 and 4 at 12.1 $\mathrm{GHz}$ are shown in Fig. 10. The currents imply that the DMs are also degenerate, and the modal radiation pattern and modal electric and magnetic fields of the first degenerate state are shown in Figs. 11 and 12, and the corresponding dynamic field figures are also uploaded to IEEE manuscript system.
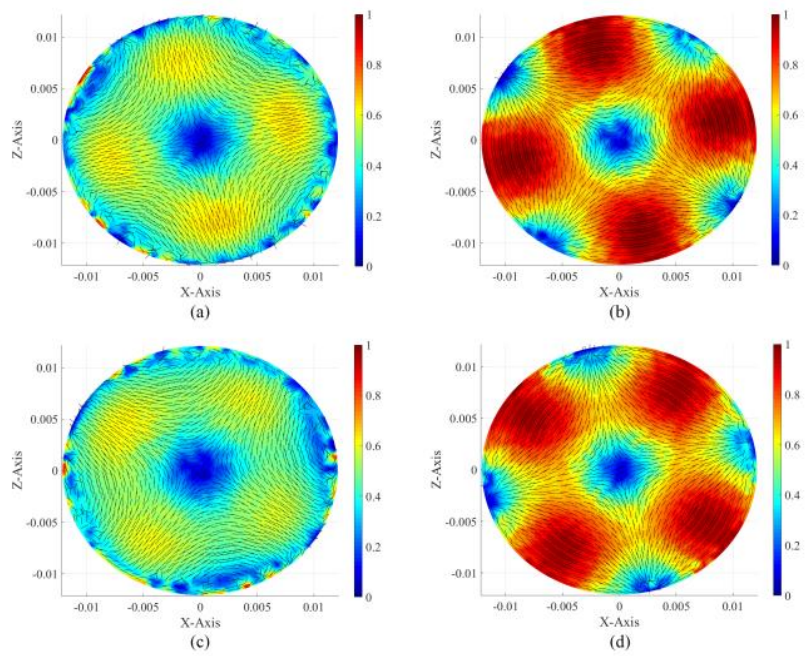

Fig. 10. (a) Modal port electric current of the resonant DM 3 at $12.1 \mathrm{GHz}$, (b) modal port magnetic current of the resonant DM 3 at $12.1 \mathrm{GHz}$, (c) modal port electric current of the resonant DM 4 at $12.1 \mathrm{GHz}$, and (d) modal port magnetic current of the resonant DM 4 at $12.1 \mathrm{GHz}$.

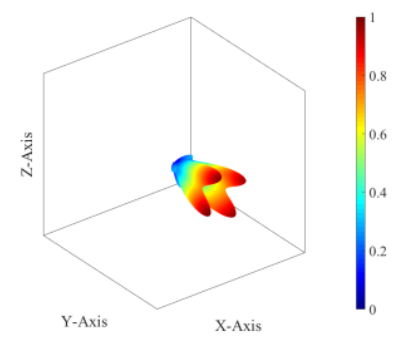

Fig. 11. Modal radiation pattern of the resonant DM 3 at $12.1 \mathrm{GHz}$.
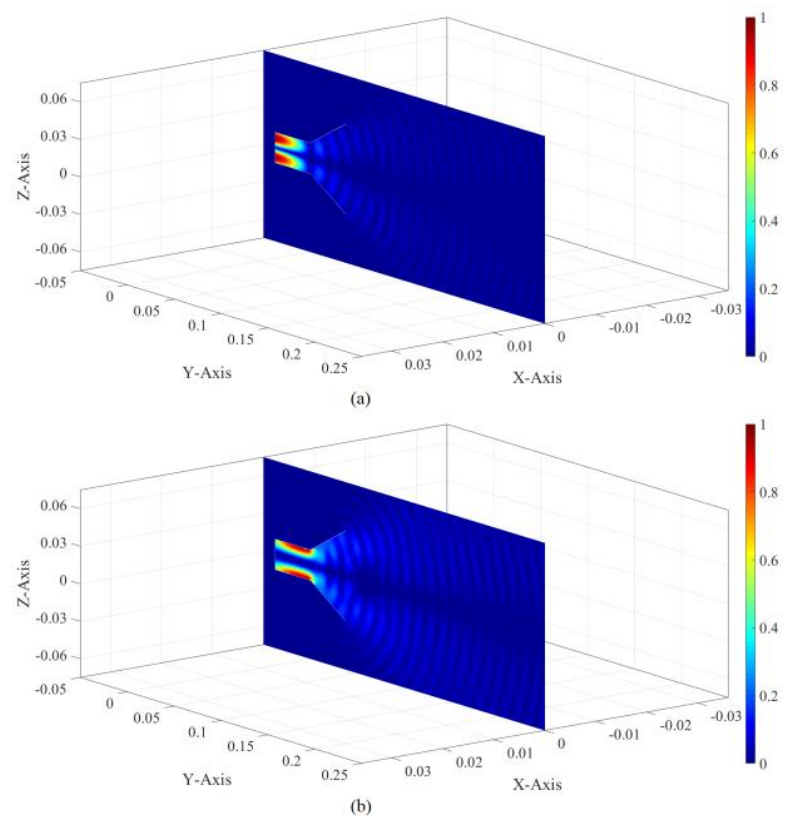

Fig. 12. Modal (a) electric and (b) magnetic field magnitude distributions of the resonant DM 3 at $12.1 \mathrm{GHz}$.

\section{Metallic Reflector Antenna}

Besides the metallic horn antenna, PTT-DMT can also be used to analyze the other kinds of wave-port-fed metallic antennas. Here, the metallic parabolic reflector antenna reported in [45] is analyzed by using PTT-DMT, and its radius and focal 
depth are $31 \mathrm{~cm}$ and $11.5 \mathrm{~cm}$ respectively, and it is fed by a wave port locating at the focal point of the reflector antenna. The JE-DoJ-based modal resistance curves are shown in Fig. 13. The figure implies that DM 1 and DM 2 are resonant at 2.72 $\mathrm{GHz}, 3.70 \mathrm{GHz}, 4.90 \mathrm{GHz}, 6.18 \mathrm{GHz}, 7.50 \mathrm{GHz}, 8.80 \mathrm{GHz}$, and $10.06 \mathrm{GHz}$.

The modal port electric currents of the resonant DMs 1 and 2 at $2.72 \mathrm{GHz}$ are shown in Fig. 14, and evidently the two modes are spatially degenerate due to the Y-axis spatially rotational symmetry of the reflector antenna, and the modal radiation pattern of the first degenerate state is shown in Fig. 15, which has a very narrow main beamwidth as expected. In addition, the modal electric and magnetic field distributions of the first degenerate state working at the resonance frequencies $2.72 \mathrm{GHz}$, $3.70 \mathrm{GHz}, 4.90 \mathrm{GHz}, 6.18 \mathrm{GHz}, 7.50 \mathrm{GHz}, 8.80 \mathrm{GHz}$, and $10.06 \mathrm{GHz}$ are shown in Fig. 16.

The modal port electric and magnetic currents, modal radiation patterns, and modal electric and magnetic field distributions of the second degenerate state are similar to the ones shown in Figs. 14-16 except a spatial rotation around Y-axis, and they are not explicitly shown here.

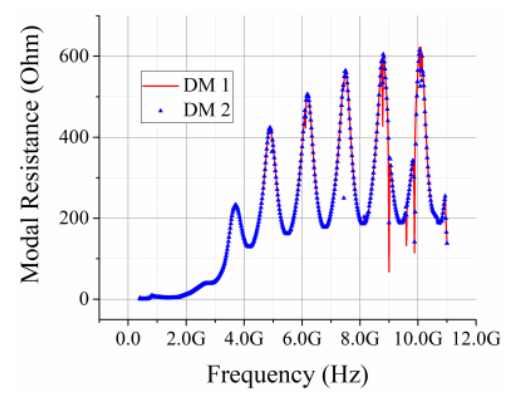

Fig. 13. JE-DoJ-based modal resistance curves of the first two lower-order DMs of the parabolic reflector antenna.
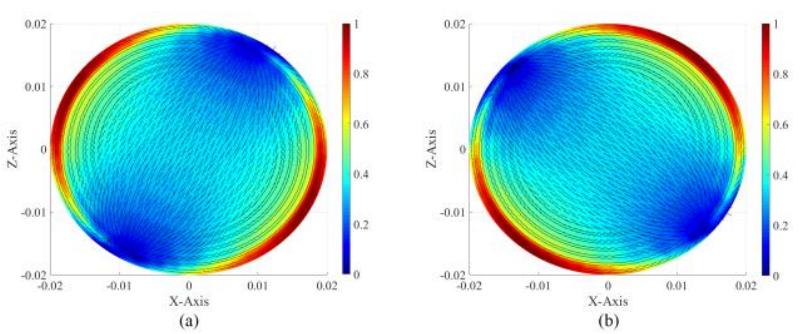

Fig. 14. Modal port electric currents of the resonant (a) DM 1 and (b) DM 2 at $2.72 \mathrm{GHz}$.

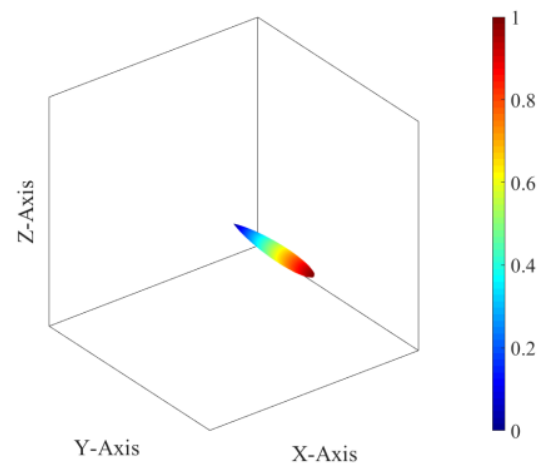

Fig. 15. Modal radiation pattern of the resonant DM 1 at $2.72 \mathrm{GHz}$.
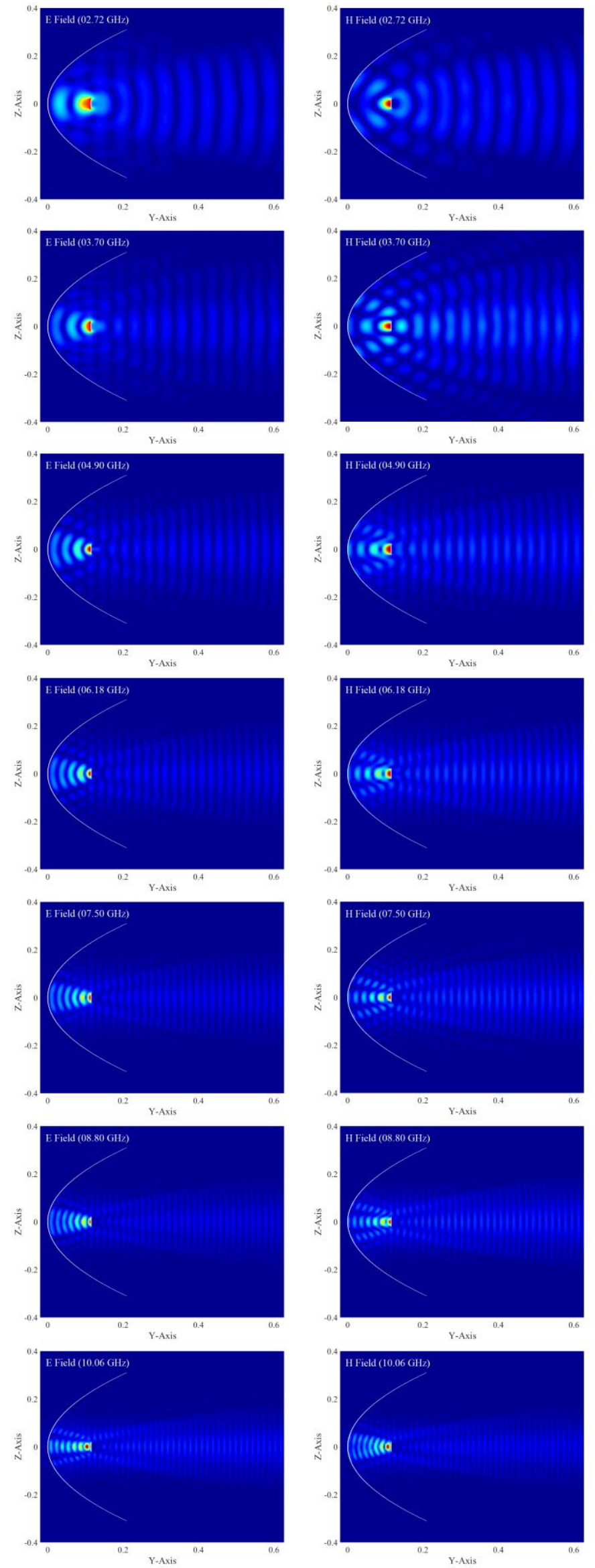

Fig. 16. Field distributions of the DM 1 working at its resonance frequencies. 
To exhibit the advantage of the novel PTT-DMT over the conventional IE-CMT, the conventional IE-CMT-based CMs of the parabolic reflector are also calculated, and the associated MSs are shown in Fig. 17. The far-field radiation patterns of the first several lower-order CMs at $2.72 \mathrm{GHz}$ are shown in Fig. 18. Evidently, the expected end-fire mode with radiation pattern Fig. 15 is not contained in the CM set. The reason leading to this phenomenon is that: the conventional IE-CMT is a modal analysis theory for scatterers, but not for antennas.

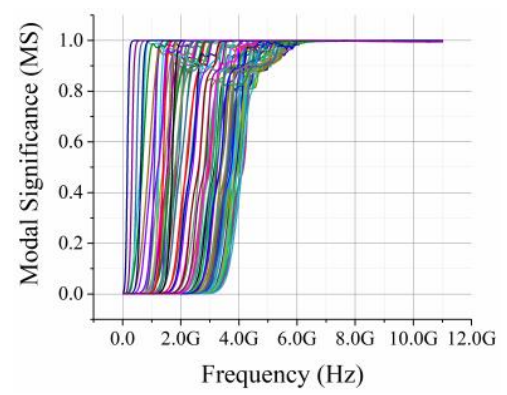

Fig. 17. MSs associated to the first several lower-order IE-CMs calculated from the conventional IE-CMT established by Harrington et al. in [8]-[10].
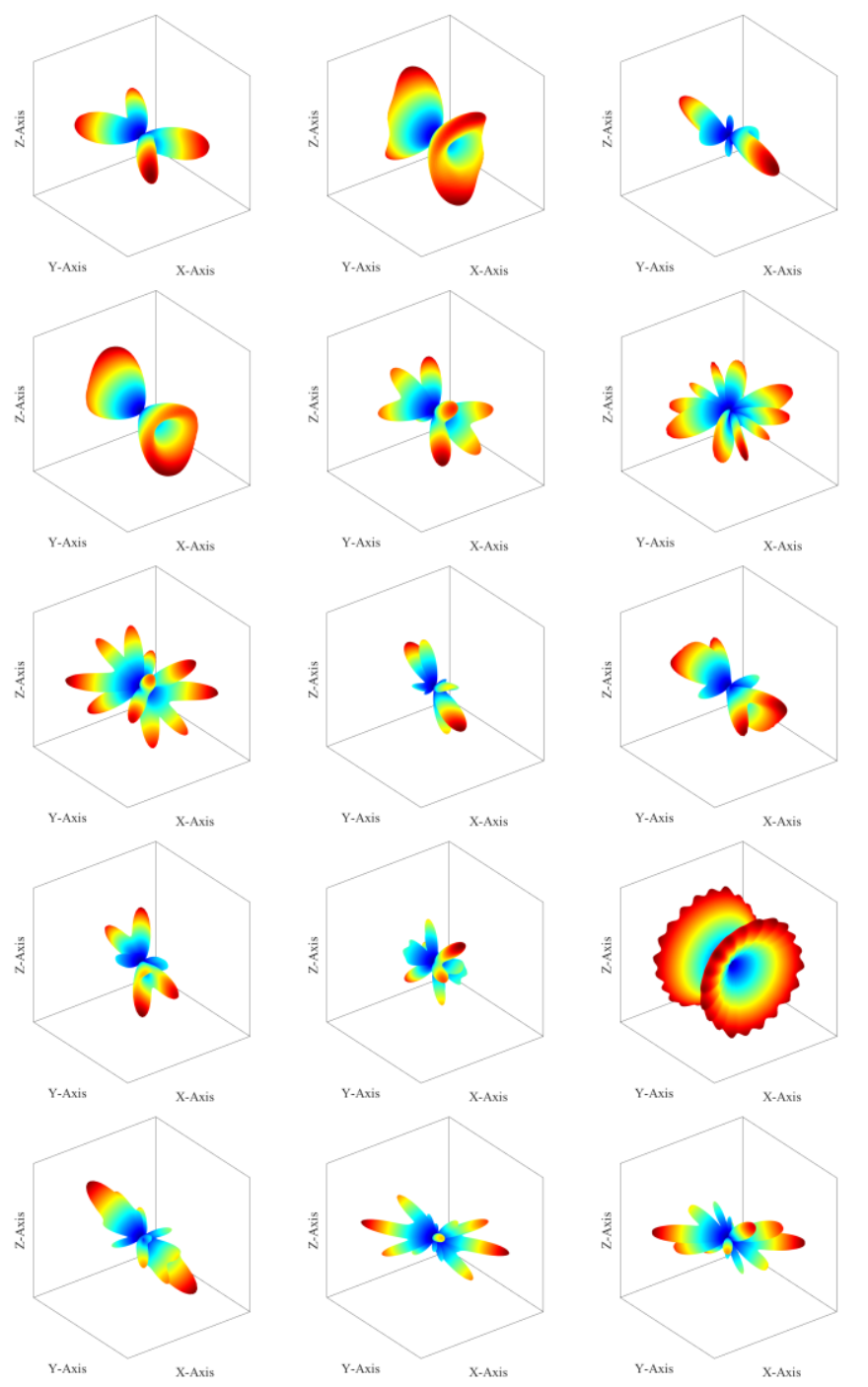

Fig. 18. Radiation patterns of the first several lower-order IE-CMs working at $2.72 \mathrm{GHz}$.

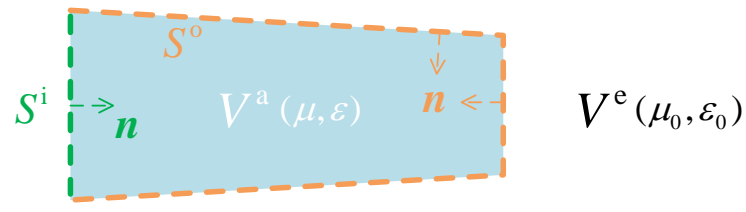

Fig. 19. Topological structure of a typical dielectric rod antenna placed in free-space environment.

\section{PTT-DMT FOR WAVE-PORT-Fed MATERIAL ANTENNAS}

Taking the dielectric rod antenna shown in Fig. 19 as a typical example, this section further generalizes the results obtained in the above Sec. II to material transmitting antennas. The dielectric rod $V^{\text {a }}$ with $(\mu, \varepsilon)$ is placed in free-space environment $V^{\mathrm{e}}$ with $\left(\mu_{0}, \varepsilon_{0}\right)$. The input port and output port of the dielectric rod are denoted as $S^{\mathrm{i}}$ and $S^{\mathrm{o}}$ respectively (obviously, $S^{\mathrm{i}} \cup S^{\mathrm{o}}$ is just the whole boundary of $V^{\mathrm{a}}$ ). The normal reference directions of $S^{\mathrm{i}}$ and $S^{\circ}$ are collectively denoted as $\boldsymbol{n}$, and $\boldsymbol{n}$ points to the interior of $V^{\text {a }}$ as shown in Fig. 19.

\section{A. Mathematical Formulations}

If the equivalent surface currents on $S^{\mathrm{i}}$ and $S^{\mathrm{o}}$ are denoted as $\left(\boldsymbol{J}^{\mathrm{i}}, \boldsymbol{M}^{\mathrm{i}}\right)$ and $\left(\boldsymbol{J}^{\mathrm{o}}, \boldsymbol{M}^{\mathrm{o}}\right)$ respectively, then the corresponding IPO can be written as follows:

$$
\begin{aligned}
P^{\text {in }} & =-(1 / 2)\left\langle\boldsymbol{J}^{\mathrm{i}},-j \omega \mu \mathcal{L}\left(\boldsymbol{J}^{\mathrm{i}}+\boldsymbol{J}^{\mathrm{o}}\right)-\mathcal{K}\left(\boldsymbol{M}^{\mathrm{i}}+\boldsymbol{M}^{\mathrm{o}}\right)\right\rangle_{S_{+}^{\mathrm{i}}} \\
& =-(1 / 2)\left\langle\boldsymbol{M}^{\mathrm{i}}, \mathcal{K}\left(\boldsymbol{J}^{\mathrm{i}}+\boldsymbol{J}^{\mathrm{o}}\right)-j \omega \varepsilon \mathcal{L}\left(\boldsymbol{M}^{\mathrm{i}}+\boldsymbol{M}^{\mathrm{o}}\right)\right\rangle_{S_{+}^{\mathrm{i}}}^{\dagger}
\end{aligned}
$$

where integral surface $S_{+}^{\mathrm{i}}$ is the right-side surface of $S^{\mathrm{i}}$. Similar to discretizing (6) into (7), integral form (23) can be discretized into the following matrix form

$$
P^{\text {in }}=\left(\mathrm{J}^{\mathrm{i}}\right)^{\dagger} \cdot \mathrm{P}_{\mathrm{JE}} \cdot\left[\begin{array}{l}
\mathrm{J}^{\mathrm{i}} \\
\mathrm{J}^{\mathrm{o}} \\
\mathrm{M}^{\mathrm{i}} \\
\mathrm{M}^{\mathrm{o}}
\end{array}\right]=\left[\begin{array}{l}
\mathrm{J}^{\mathrm{i}} \\
\mathrm{J}^{\mathrm{o}} \\
\mathrm{M}^{\mathrm{i}} \\
\mathrm{M}^{\mathrm{o}}
\end{array}\right]^{\dagger} \cdot \mathrm{P}_{\mathrm{HM}} \cdot \mathrm{M}^{\mathrm{i}}
$$

which includes both independent current $\mathrm{J}^{\mathrm{i}} / \mathrm{M}^{\mathrm{i}}$ and dependent currents $\left(J^{\circ}, M^{i}, M^{\circ}\right) /\left(J^{i}, J^{o}, M^{\circ}\right)$.

Using a similar method to Sec. II-B, the transformations from independent current into dependent currents can be derived, and the detailed derivation is provided in the appendix of this paper. Inserting the transformations into (24), the IPO $P^{\text {in }}=\left(\mathrm{C}^{\mathrm{i}}\right)^{\dagger} \cdot \mathrm{P}^{\text {in }} \cdot \mathrm{C}^{\mathrm{i}}$ with only independent current $\mathrm{C}^{\mathrm{i}}$ (which is either $\mathrm{J}^{\mathrm{i}}$ or $\mathrm{M}^{\mathrm{i}}$ ) is derived. By decomposing $\mathrm{P}^{\text {in }}$ into its Hermitian parts $\mathrm{P}_{+}^{\text {in }}$ and $\mathrm{P}_{-}^{\text {in }}$, the DMs can be derived from solving modal decoupling equation $\mathrm{P}_{-}^{\text {in }} \cdot \mathrm{C}_{m}^{\mathrm{i}}=\theta_{m} \mathrm{P}_{+}^{\mathrm{in}} \cdot \mathrm{C}_{m}^{\mathrm{i}}$.

\section{B. Numerical Verifications}

In this subsection, the PTT-DMT is employed to do the modal analysis for the dielectric rod antenna reported in [46], and the antenna is a circular cone with base radius $13 \mathrm{~mm}$, top radius $9 \mathrm{~mm}$, height $90 \mathrm{~mm}$, and material parameters $\mu_{\mathrm{r}}=1$, $\varepsilon_{\mathrm{r}}=2.2$, and $\sigma=0$. Here, the base is selected as input port. 
The HM-DoM-based modal resistance curves of the dielectric rod antenna are shown in Fig. 20. Clearly, the DM 1 and DM 2 are resonant at $8.46 \mathrm{GHz}, 9.50 \mathrm{GHz}, 10.54 \mathrm{GHz}$ and $11.58 \mathrm{GHz}$, and the DM 3 is resonant at $9.36 \mathrm{GHz}, 10.24 \mathrm{GHz}$ and $11.22 \mathrm{GHz}$.

For the resonant DM 1 and DM 2 at $8.46 \mathrm{GHz}$, their modal port currents are shown in Fig. 21, and the figure implies that the modes are degenerate spatially due to the rotational symmetry around $\mathrm{Y}$-axis. The modal radiation pattern of the resonant DM 1 at $8.46 \mathrm{GHz}$ is shown in Fig. 22. The modal field distributions of the resonant DM 1 at its a series of resonance frequencies are shown in Fig. 23.

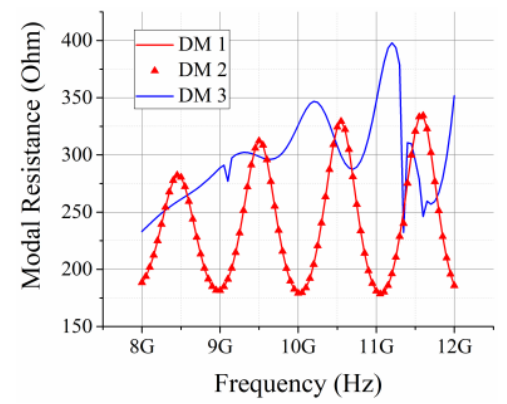

Fig. 20. HM-DoM-based modal resistances of the first three lower-order DMs of the dielectric rod antenna.

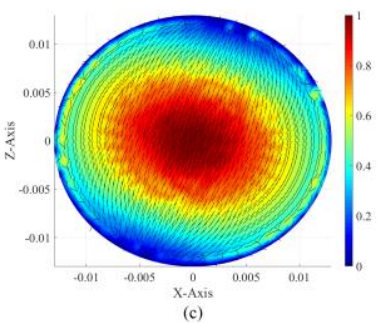

(c)

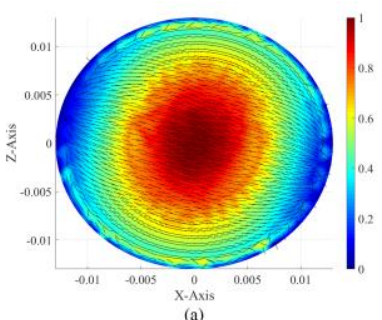

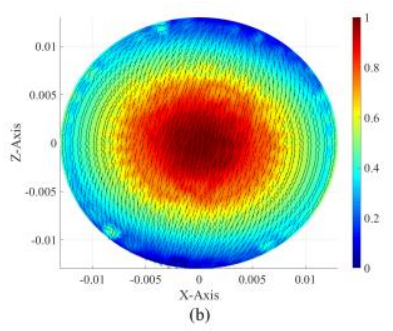

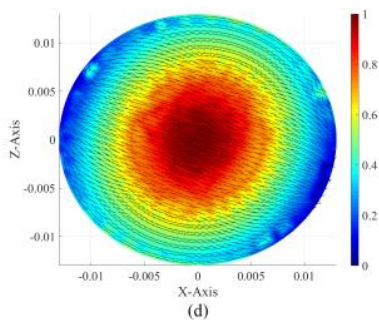

Fig. 21. (a) Modal port electric current of the resonant DM 1 at $8.46 \mathrm{GHz}$, (b) modal port magnetic current of the resonant DM 1 at $8.46 \mathrm{GHz}$, (c) modal port electric current of the resonant DM 2 at $8.46 \mathrm{GHz}$, and (d) modal port magnetic current of the resonant DM 2 at $8.46 \mathrm{GHz}$.

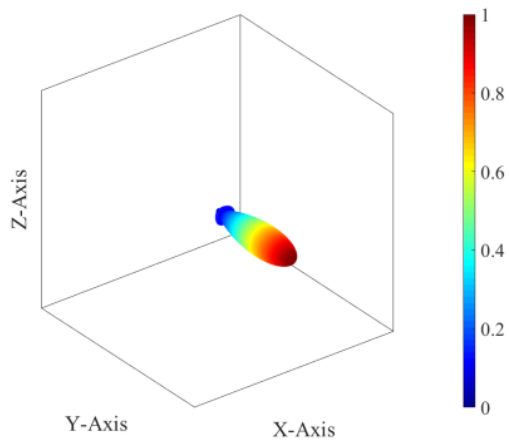

Fig. 22. Modal radiation pattern of the resonant DM 1 at $8.46 \mathrm{GHz}$.
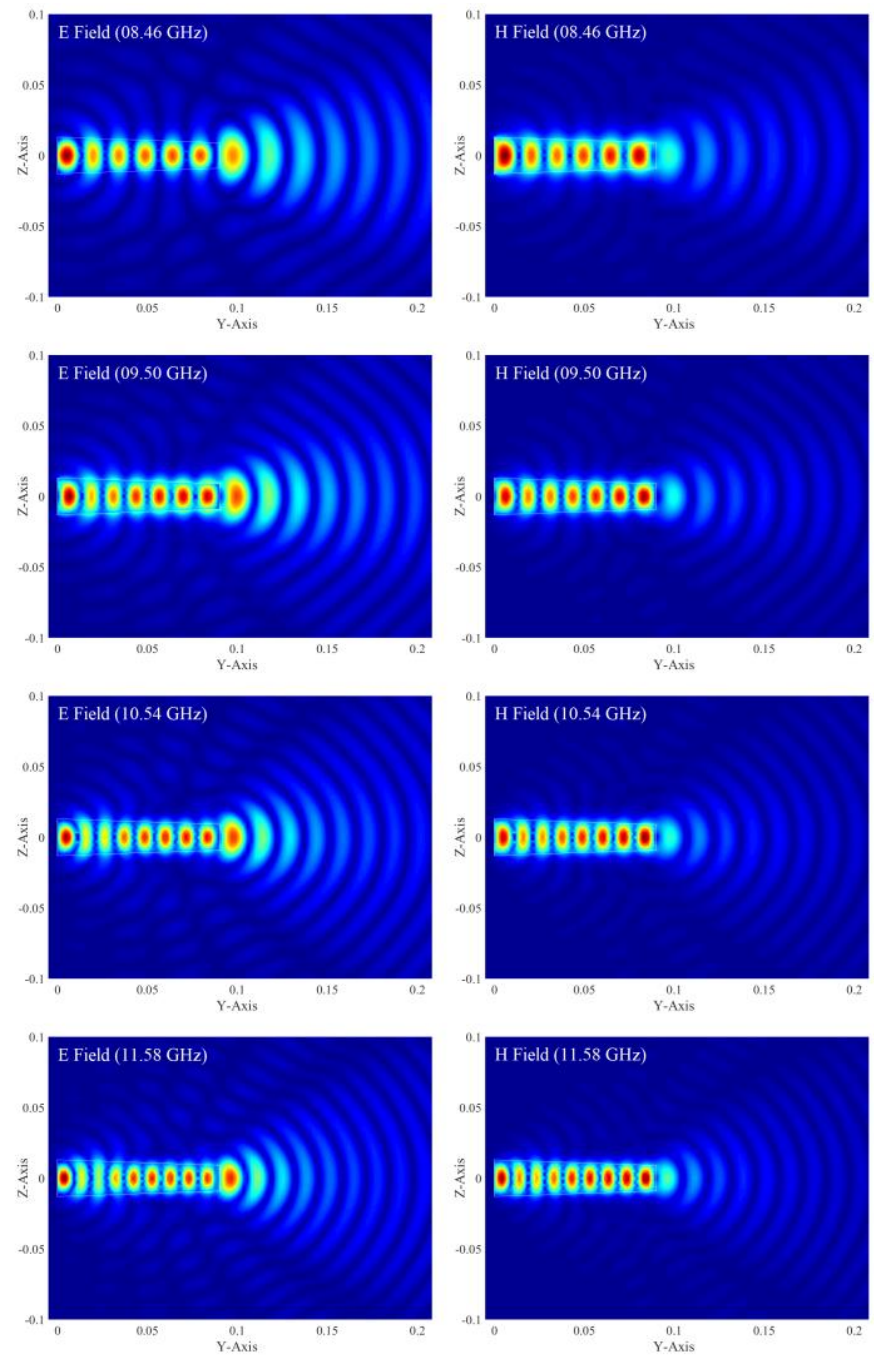

Fig. 23. Modal electric and magnetic field distributions of the DM 1 working at its a series of resonance frequencies.
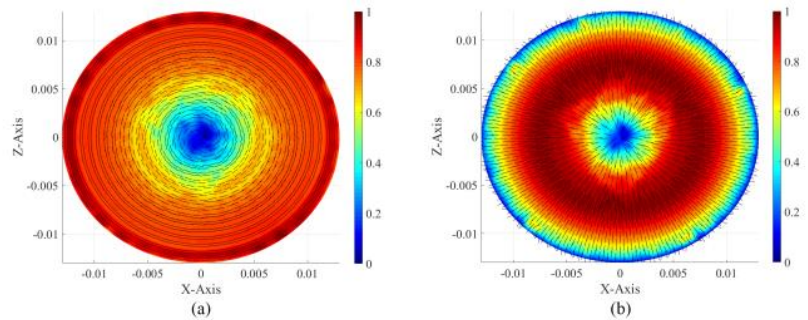

Fig. 24. Modal port (a) electric and (b) magnetic currents of the resonant DM 3 working at $9.36 \mathrm{GHz}$.

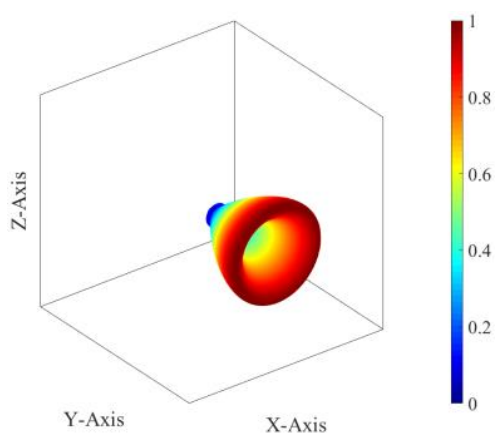

Fig. 25. Modal radiation pattern of the resonant DM 3 at $9.36 \mathrm{GHz}$. 

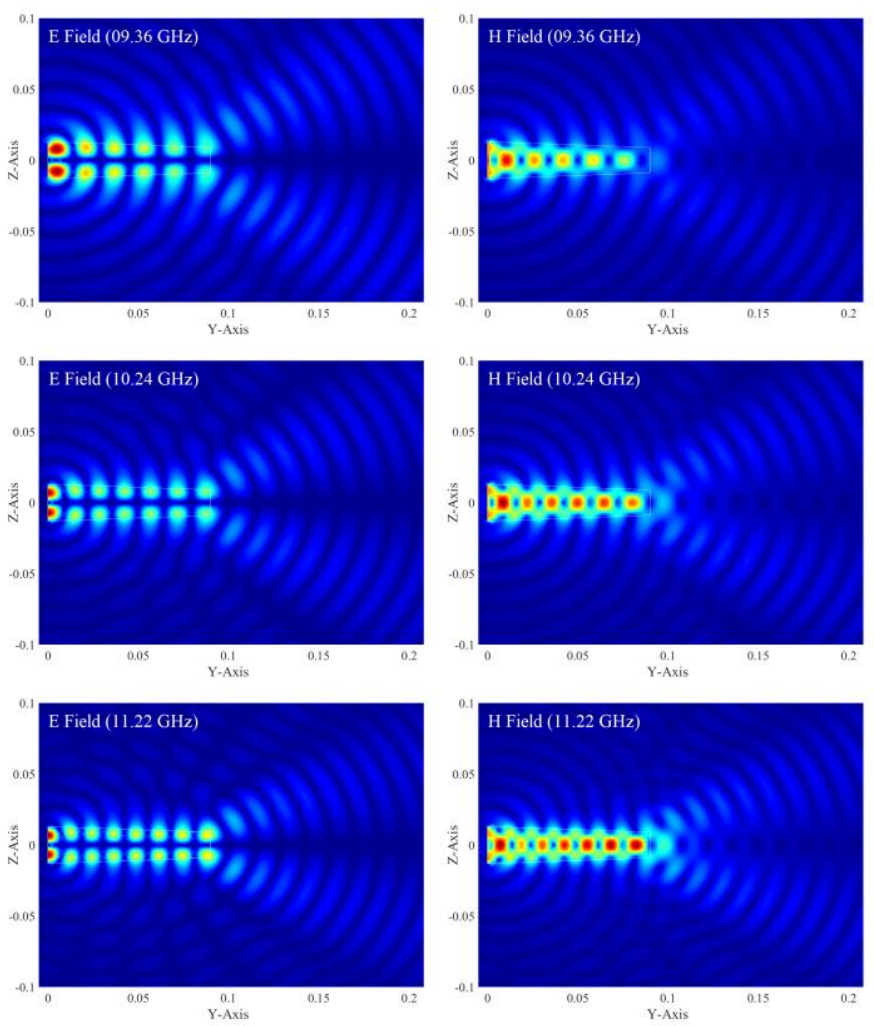

Fig. 26. Modal electric and magnetic field distributions of the DM 3 working at its a series of resonance frequencies.

For the resonant DM 3 at $9.36 \mathrm{GHz}$, its modal port currents are shown in Fig. 24. The Fig. 24 implies that the mode is rotationally symmetrical around $\mathrm{Y}$-axis, so the mode has only one degenerate state. The modal radiation pattern of the resonant DM 3 at $9.36 \mathrm{GHz}$ is shown in Fig. 25. The modal field distributions of the resonant DM 3 at its resonance frequencies are shown in Fig. 26.

To exhibit the advantage of the novel PTT-DMT over the conventional IE-CMT, the conventional IE-CMT-based CMs of the dielectric rod are also calculated, and the associated MSs are shown in Fig. 27. The far-field radiation patterns of the first several lower-order CMs at $8.46 \mathrm{GHz}$ are shown in Fig. 28. Evidently, the expected end-fire mode with radiation pattern Fig. 22 is not contained in the CM set, because the conventional IE-CMT is a modal analysis theory for scattering objects rather than for transmitting antennas.

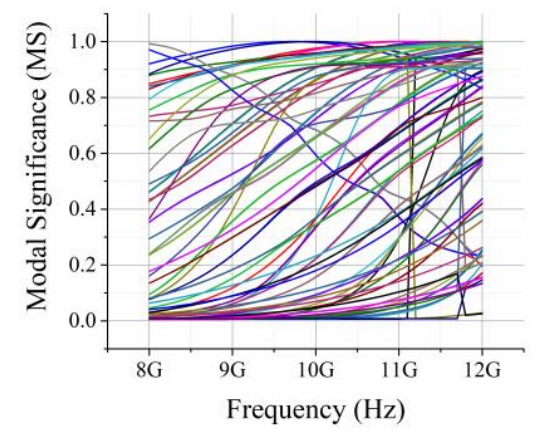

Fig. 27. MSs associated to the first several lower-order IE-CMs calculated from the conventional IE-CMT established by Harrington et al. in [11]-[12].

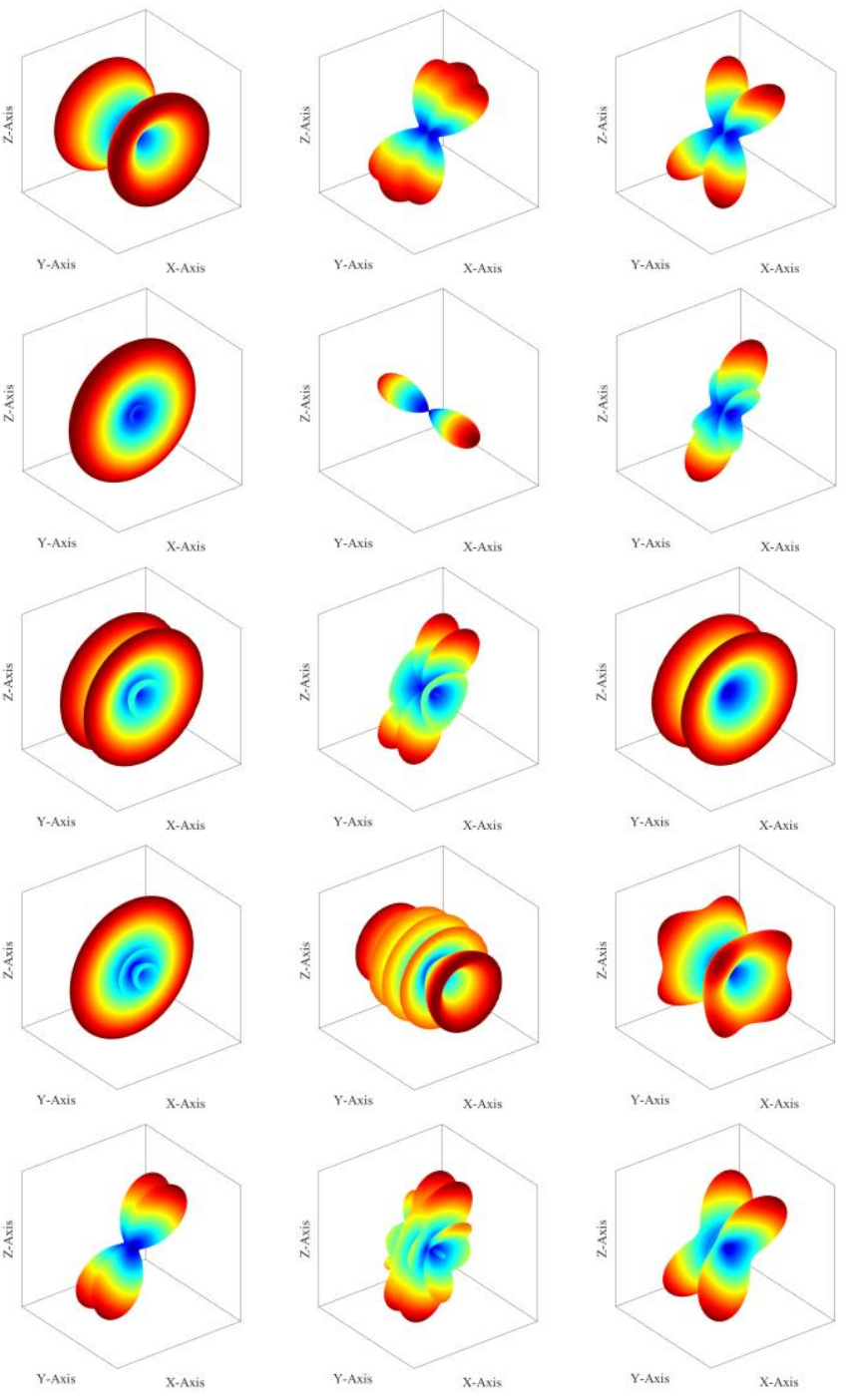

Fig. 28. Radiation patterns of several lower-order IE-CMs at $8.46 \mathrm{GHz}$.

\section{CONCLUDING REMARKS}

Incident-field-driven scattering object, lumped-port-driven transmitting antenna, and wave-port-fed transmitting antenna are three different kinds of EM structures. The difference between the scattering object and two transmitting antennas is mainly reflected in their working mechanisms. The difference between the lumped-port-driven transmitting antenna and the wave-port-fed transmitting antenna is mainly reflected in their excitation manners. The differences imply that these different EM structures need different modal analysis methods.

The physical interpretations for the existing various CMTs expose the following impotent facts:

1) the SM-CMT built in [5]-[7], the IE-CMT built in [8]-[12], and the WEP-CMT built in [13]-[15] are the modal analysis theories for scattering objects;

2) the WEP-CMT built in [35] and [36, Sec. 4.2] is a modal analysis theory for lumped-port-driven transmitting antennas;

3) the existing CMT-based modal analysis and designs for wave-port-fed transmitting antennas are approximate but not rigorous. 
This paper is devoted to establishing a novel and rigorous modal analysis theory - DMT - for wave-port-fed transmitting antennas, and constructing the corresponding fundamental orthogonal modes - DMs - of a pre-selected wave-port-fed transmitting antenna.

It is found out that: the PTT quantifying the power transport process related to the objective antenna is a proper framework for establishing DMT; the IPO as the source term in PTT is a reasonable modal generating operator; the dependent variable elimination (DVE) similar to the one widely used in CMT is an indispensable process for eliminating the dependent currents in IPO; the modal decoupling equation with a similar mathematical form to the conventional characteristic equation is an effective equation for calculating DMs.

The general steps for constructing the DMs are summarized as follows:

Step 1. Decomposing the boundaries of objective antenna;

Step 2. Deriving PTT;

Step 3. Formulating IPO and discretizing it into matrix form;

Step 4. Eliminating the dependent currents in IPO;

Step 5. Establishing and solving modal decoupling equation.

The DMs constructed above don't have net energy exchange in any integral period, and satisfy Parseval's identity.

Modal electric-magnetic energy-decoupling factor / modal electric-magnetic phase-mismatching factor (modal $\Theta$-factor) is a quantitative description for the decoupling / mismatching degree between the electric and magnetic energies / phases of a modal field. A field-based definition for modal input impedance and admittance can be selected as a replacement for the conventional circuit-based definition, and the resonance frequencies of DMs can be effectively recognized by finding the local maximums of the field-based modal resistance or conductance curves. The frequently used modal quantity MS can be physically interpreted in three somewhat different but not contradictory ways, and they are

1) MS is a quantitative depiction for the weight of a certain DM in whole DM-based modal expansion formulation;

2) for a single DM, MS is a quantitative depiction for the weight of the modal input resistance/conductance in whole modal input impedance/admittance;

3) MS is a quantitative depiction for the coupling/matching degree of the electric and magnetic fields carried by a single DM.

By using both the novel PTT-DMT and the conventional CMT to analyze some classical transmitting antennas, the validities of PTT-DMT are verified, and the advantages of PTT-DMT are exhibited. In addition, the PTT-DMT for wave-port-fed transmitting antennas will be further generalized to wave-port-fed wave-guiding structures in our future studies, such that the modal analysis for transmitting antennas and their feeding structures can be done in a unified physical framework - PTT.

\section{APPENDIX}

Some detailed formulations related to Sec. III-A are provided as below.
The transformation from independent current $\mathrm{J}^{\mathrm{i}} / \mathrm{M}^{\mathrm{i}}$ into dependent currents $\left(J^{0}, M^{i}, M^{0}\right) /\left(J^{i}, J^{0}, M^{\circ}\right)$ is based on the following integral equations

$$
\begin{aligned}
& {\left[\mathcal{K}\left(\boldsymbol{J}^{\mathrm{i}}+\boldsymbol{J}^{\mathrm{o}}\right)-j \omega \varepsilon \mathcal{L}\left(\boldsymbol{M}^{\mathrm{i}}+\boldsymbol{M}^{\mathrm{o}}\right)\right]_{S_{+}^{\mathrm{i}}}^{\mathrm{tan}}=\boldsymbol{J}^{\mathrm{i}} \times \boldsymbol{n} } \\
& {\left[-j \omega \mu \mathcal{L}\left(\boldsymbol{J}^{\mathrm{i}}+\boldsymbol{J}^{\mathrm{o}}\right)-\mathcal{K}\left(\boldsymbol{M}^{\mathrm{i}}+\boldsymbol{M}^{\mathrm{o}}\right)\right]_{S_{+}^{\mathrm{i}}}^{\mathrm{tan}}=\boldsymbol{n} \times \boldsymbol{M}^{\mathrm{i}} } \\
& {\left[-j \omega \mu \mathcal{L}\left(\boldsymbol{J}^{\mathrm{i}}+\boldsymbol{J}^{\mathrm{o}}\right)-\mathcal{K}\left(\boldsymbol{M}^{\mathrm{i}}+\boldsymbol{M}^{\mathrm{o}}\right)\right]_{S_{-}^{\mathrm{o}}}^{\text {tan }} } \\
= & {\left[-j \omega \mu_{0} \mathcal{L}_{0}\left(-\boldsymbol{J}^{\mathrm{o}}\right)-\mathcal{K}_{0}\left(-\boldsymbol{M}^{\mathrm{o}}\right)\right]_{S_{+}^{\mathrm{o}}}^{\mathrm{tan}} } \\
& {\left[\mathcal{K}\left(\boldsymbol{J}^{\mathrm{i}}+\boldsymbol{J}^{\mathrm{o}}\right)-j \omega \varepsilon \mathcal{L}\left(\boldsymbol{M}^{\mathrm{i}}+\boldsymbol{M}^{\mathrm{o}}\right)\right]_{S_{-}^{\mathrm{o}}}^{\tan } } \\
= & {\left[\mathcal{K}_{0}\left(-\boldsymbol{J}^{\mathrm{o}}\right)-j \omega \varepsilon_{0} \mathcal{L}_{0}\left(-\boldsymbol{M}^{\mathrm{o}}\right)\right]_{S_{+}^{\mathrm{o}}}^{\mathrm{tan}} }
\end{aligned}
$$

Here, (25) and (26) originate from the definitions for $\boldsymbol{J}^{\mathrm{i}}$ and $\boldsymbol{M}^{\mathrm{i}}$ respectively; (27) and (28) originate from the tangential electric and magnetic field continuation conditions on $S^{\circ}$ respectively, where integral surfaces $S_{-}^{\circ}$ and $S_{+}^{\circ}$ are the inner-side and outer-side surfaces of $S^{\circ}$ respectively.

\section{REFERENCES}

[1] P. M. Morse and H. Feshbach, Methods of Theoretical Physics. New York: McGraw-Hill, 1953.

[2] R. Courant and D. Hilbert, Methods of Mathematical Physics. New York: Wiley, 1989.

[3] E. C. Titchmarsh, Eigenfunction Expansions Associated with Second-order Differential Equations, 2nd. London: Oxford University Press, 1962.

[4] J. Jackson, Classical Electrodynamics, 3rd. New York: Wiley, 1999.

[5] R. J. Garbacz, "Modal expansions for resonance scattering phenomena," Proc. IEEE, vol. 53, no. 8, pp. 856-864, Aug. 1965.

[6] R. J. Garbacz, "A generalized expansion for radiated and scattered fields," Ph.D. dissertation, Dept. Elect. Eng., The Ohio State Univ., Columbus, OH, USA, 1968.

[7] R. J. Garbacz and R. H. Turpin, "A generalized expansion for radiated and scattered fields," IEEE Trans. Antennas Propag., vol. AP-19, no. 3, pp. 348-358, May 1971.

[8] R. F. Harrington and J. R. Mautz, Theory and Computation of Characteristic Modes for Conducting Bodies. Interaction Notes, Note 195, Syracuse Univ., Syracuse, New York, USA, Dec. 1970.

[9] R. F. Harrington and J. R. Mautz, "Theory of characteristic modes for conducting bodies," IEEE Trans. Antennas Propag., vol. AP-19, no. 5, pp. 622-628, Sep. 1971.

[10] R. F. Harrington and J. R. Mautz, "Computation of characteristic modes for conducting bodies," IEEE Trans. Antennas Propag., vol. AP-19, no. 5, pp. 629-639, Sep. 1971.

[11] R. F. Harrington, J. R. Mautz, and Y. Chang, "Characteristic modes for dielectric and magnetic bodies," IEEE Trans. Antennas Propag., vol. AP-20, no. 2, pp. 194-198, Mar. 1972.

[12] Y. Chang and R. F. Harrington, "A surface formulation for characteristic modes of material bodies," IEEE Trans. Antennas Propag., vol. 25, no. 6, pp. 789-795, Nov. 1977.

[13] R. Z. Lian, "Research on the work-energy principle based characteristic mode theory for scattering systems," Doctoral Dissertation, School of Electronic Science and Engineering, University of Electronic Science and Technology of China (UESTC), Chengdu, Sichuan, China, July 22, 2019. [Online]. Available: https://arxiv.org/abs/1907.11787.

[14] R. Z. Lian, X. Y. Guo, and M. Y. Xia, "Work-energy principle based characteristic mode theory with solution domain compression for material scattering systems," with IEEE-TAP Manuscript Identifier 
[AP2004-0708] submitted to IEEE Trans. Antennas Propag. on 12-Apr-2020.

[15] R. Z. Lian, X. Y. Guo, and M. Y. Xia, "Work-energy principle based characteristic mode theory with solution domain compression for metal-material composite scattering systems," with IEEE-TAP Manuscript Identifier [AP2004-0709] submitted to IEEE Trans. Antennas Propag. on 12-Apr-2020.

[16] J.-F. Lin and L. Zhu, "Low-profile high-directivity circularly-polarized differential-fed patch antenna with characteristic mode analysis," IEEE Trans. Antennas Propag., vol. 69, no. 2, pp. 723-733, Feb. 2021.

[17] X. Gao, G. W. Tian, Z. Y. Shou, and S. M. Li, "A low-profile broadband circularly polarized patch antenna based on characteristic mode analysis," IEEE Antennas Wireless Propag. Lett., vol. 20, no. 2, pp. 214-218, Feb. 2021.

[18] K. A. Obeidat, B. D. Raines, R. G. Rojas, and B. T. Strojny, "Design of frequency reconfigurable antennas using the theory of network characteristic modes," IEEE Trans. Antennas Propag., vol. 58, no. 10, pp. 3106-3113, Oct. 2010.

[19] L. Zhang, Y. H. Sun, Y. J. He, S.-W. Wong, C. X. Mao, L. Ge, and S. Gao, "A quad-polarization reconfigurable antenna with suppressed cross polarization based on characteristic mode theory," IEEE Trans. Antennas Propag., vol. 69, no. 2, pp. 636-647, Feb. 2021.

[20] S. L. Cao, Z. Zhang, X. N. Fu, and J. H. Wang, "Pattern-reconfigurable bidirectional antenna design using the characteristic mode analysis," IEEE Antennas Wireless Propag. Lett., vol. 20, no. 1, pp. 53-57, Jan. 2021.

[21] G. P. Gao, R.-F. Zhang, W.-F. Geng, H.-J. Meng, and B. Hu, "Characteristic mode analysis of a nonuniform metasurface antenna for wearable applications," IEEE Antennas Wireless Propag. Lett., vol. 19, no. 8, pp. 1355-1359, Aug. 2020.

[22] Y. Yan, J. Ouyang, X. Ma, R. Wang, and A. Sharif, "Circularly polarized RFID tag antenna design for metallic poles using characteristic mode analysis," IEEE Antennas Wireless Propag. Lett., vol. 18, no. 7, pp. 1327-1331, Jul. 2019.

[23] A. Sharif, J. Ouyang, F. Yang, H. T. Chattha, M. A. Imran, A. Alomainy, and Q. H. Abbasi, "Low-cost inkjet-printed UHF RFID tag-based system for internet of things applications using characteristic modes," IEEE Internet Thing J., vol. 6, no. 2, pp. 3962-3975, Apr. 2019.

[24] R. Y. Ma and N. Behdad, "Design of platform-based HF direction-finding antennas using the characteristic mode theory," IEEE Trans. Antennas Propag., vol. 67, no. 3, pp. 1417-1427, Mar. 2019.

[25] N. Peitzmeier and D. Manteuffel, "Upper bounds and design guidelines for realizing uncorrelated ports on multimode antennas based on symmetry analysis of characteristic modes," IEEE Trans. Antennas Propag., vol. 67, no. 6, pp. 3902-3914, Jun. 2019.

[26] W. Su, Q. Y. Zhang, S. Alkaraki, Y. Zhang, X.-Y. Zhang, and Y. Gao, "Radiation energy and mutual coupling evaluation for multimode MIMO antenna based on the theory of characteristic mode," IEEE Trans. Antennas Propag., vol. 67, no. 1, pp. 74-84, Jan. 2019.

[27] M. Cabedo-Fabres, E. Antonino-Daviu, and A. Valero-Nogueira, et al., "The theory of characteristic modes revisited: a contribution to the design of antennas for modern applications," IEEE Antennas Propag. Mag., vol. 49, no. 5, pp. 52-68, Oct. 2007.

[28] M. Vogel, G. Gampala, and D. Ludick, et al., "Characteristic mode analysis: putting physics back into simulation," IEEE Antennas Propag. Mag., vol. 57, no. 2, pp. 307-317, Apr. 2015.

[29] Y. K. Chen and C.-F. Wang, Characteristic Modes: Theory and Applications in Antenna Engineering. Hoboken: Wiley, 2015.

[30] J. Chalas, K. Sertel, and J. L. Volakis, "Computation of the Q limits for arbitrary-shaped antennas using characteristic modes," IEEE Trans. Antennas Propag., vol. 64, no. 7, pp. 2637-2647, Jul. 2016.

[31] M. Khan and D. Chatterjee, "Analysis of reactive loading in a U-slot microstrip patch using the theory of characteristic modes [antenna applications corner]," IEEE Antennas Propag. Mag., vol. 60, no. 6, pp. 88-97, Dec. 2018.

[32] L. Guan, Z. He, D. Z. Ding, and R. S. Chen, "Efficient characteristic mode analysis for radiation problems of antenna arrays," IEEE Trans. Antennas Propag., vol. 67, no. 1, pp. 199-206, Jan. 2019.

[33] Q. Wu, "Characteristic mode assisted design of dielectric resonator antennas with feedings," IEEE Trans. Antennas Propag., vol. 67, no. 8, pp. 5294-5304, Aug. 2019.

[34] Q. Wu, "General metallic-dielectric structures: a characteristic mode analysis using volume-surface formulations," IEEE Antennas Propag. Mag., vol. 61, no. 3, pp. 27-36, Jun. 2019.
[35] R. Z. Lian, M. Y. Xia, and X. Y. Guo, "Work-energy principle based characteristic mode analysis for Yagi-Uda arrays," with IEEE-TAP Manuscript Identifier [AP2101-0036] submitted to IEEE Trans. Antennas Propag. on 06-Jan-2021.

[36] R. Z. Lian, "Energy-viewpoint-based electromagnetic modal analysis," Post-Doctoral Concluding Report, Department of Electronics, School of Electronics Engineering and Computer Science, Peking University (PKU), Beijing, China, August 7, 2021. [Online]. Available: https://arxiv.org/abs/2108.03432.

[37] IEEE, The Authoritative Dictionary of IEEE Standards Terms, 7th. New York: IEEE Press, 2000.

[38] R. Z. Lian, M. Y. Xia, and X. Y. Guo, "Work-energy principle based characteristic mode theory for wireless power transfer systems," with IEEE-TAP Manuscript Identifier [AP2101-0035] submitted to IEEE Trans. Antennas Propag. on 06-Jan-2021. [Online]. Available: https://www.techrxiv.org/articles/preprint/Work-Energy_Principle_Base d_Characteristic_Mode_Theory_for_Wireless_Power_Transfer_Systems $/ 15134052$.

[39] R. Z. Lian and M. Y. Xia, "Power transport theorem based decoupling mode theory for wave-guiding structures," with IEEE-TMTT Manuscript Identifier [TMTT-2021-04-0464] submitted to IEEE Trans. Microw. Theory Techn. on 14-Apr-2021. [Online]. Available: https://www.techrxiv.org/articles/preprint/Power_Transport_Theorem_B ased_Decoupling_Mode_Theory_for_Wave-guiding_Structures/1514 $\overline{5} 1$ 97.

[40] R. Z. Lian, "Research on the power transport theorem based decoupling mode theory for transceiving systems," Post-Doctoral Research Report, Department of Electronics, School of Electronics Engineering and Computer Science, Peking University (PKU), Beijing, China, February 11, 2021. [Online]. Available: https://arxiv.org/abs/2103.01853.

[41] C. A. Balanis et al., Modern Antenna Handbook. Hoboken: Wiley, 2008.

[42] R. P. Feynman, R. B. Leighton, and M. Sands, The Feynman Lectures on Physics, Vol. II. New York: Addison-Wesley Publishing Company, 1964.

[43] R. Z. Lian and J. Pan, "Electromagnetic-power-based modal classification, modal expansion, and modal decomposition for perfect electric conductors," Int. J. Antennas Propag., vol. 2018, pp. 1-17, Nov. 2018.

[44] Y.-X. Guo, K.-M. Luk, and K.-W. Leung, "Characteristics of aperture-coupled cylinderical dielectric resonantor antennas on a thick ground plane," Inst. Elect. Eng. Proc. Microwave Antennas Propagat., vol. 146, no. 6, pp. 439-442, Dec. 1999.

[45] S. I. Ghobrial and H. R. Sharobim, "Radiation patterns of a paraboloidal reflector fed by a pyramidal horn with lossy walls," IEEE Trans. Antennas Propag., vol. 37, no. 10, pp. 1316-1317, Oct. 1989.

[46] K. A. S. Qassim and N. J. McEwan, "Focal plane dielectric rod arrays with printed launches," in 1993 Eighth International Conference on Antennas and Propagation, Edinburgh, UK, Mar. 1993, pp.792-795. 